\title{
THE ENUMERATIVE GEOMETRY OF PLANE CUBICS. I: SMOOTH CUBICS
}

\author{
PAOLO ALUFFI
}

\begin{abstract}
We construct a variety of complete plane cubics by a sequence of five blow-ups over $\mathbf{P}^{9}$. This enables us to translate the problem of computing characteristic numbers for a family of plane cubics into one of computing five Segre classes, and to recover classic enumerative results of Zeuthen and Maillard.
\end{abstract}

\section{INTRODUCTION}

This paper is devoted to the computation of the characteristic numbers for the 9-dimensional family of smooth plane cubics, i.e. the number of nonsingular plane cubics which are tangent to $n_{l}$ lines and contain $9-n$, points in general position in the plane. We plan to complement this result with the computation of the characteristic numbers for nodal and cuspidal cubics, in a forthcoming paper.

Classically, the enumerative geometry of plane cubics was studied independently by S. Maillard and H. S. Zeuthen around 1870 [M, Z]; their results also appear in [Sc, Chapter 4, §24]. However, as with many other accomplishments of the great enumerative geometers of the nineteenth century, the rigor of the methods used in $[\mathrm{M}, \mathrm{Z}]$ was soon questioned. In the past few years interest in enumerative geometry has revived, partially as a consequence of a new and deeper understanding of intersection theory, and in many cases the old results and methods have been verified, improved, or corrected. In the case of plane cubics, there are already several modern approaches partially verifying Maillard and Zeuthen's results (see [Sa, KS, XM]). In general, these approaches work in the vein of the classic "degeneration method": by specializing the families to more degenerate ones, and using previously obtained results. Kleiman and Speiser, in particular, have developed an efficient procedure of "partially" compactifying the family under examination. They normalize part of the graph of the dual map, in such a way that the elementary systems (the basic tool for relating the characteristic numbers of different families) are incorporated as complete subschemes.

Received by the editors October 24, 1988.

1980 Mathematics Subject Classification (1985 Revision). Primary 14N10; Secondary 14C17.

Key words and phrases. Complete conics, complete cubics, blow-up, Segre class. 
We present here an approach with a different flavor. We dominate the graph of the dual map with a nonsingular variety $\widetilde{V}$, which is obtained from the $\mathbb{P}^{9}$ parametrizing plane cubics by a sequence of blow-ups, in the spirit of (for example) Veinsencher's spaces of 'complete quadrics' [V]. Also, rather than employing the method of degeneration, we reduce the computation of the numbers for a family of reduced cubics to the computation of certain Segre classes related to the behavior of the family in the blow-up process. This choice, which has its roots in Fulton-Mac Pherson's 'static' intersection theory, forces us to an extensive analysis of the blow-ups -indeed, this choice forces us to desingularize the whole graph; its net advantage is that a specific family can be studied without dependence on other results. The characteristic numbers for smooth cubics, for example, are an immediate by-product of our construction of $\widetilde{V}$, while their computation via the degeneration method relies on sophisticated information about families of singular cubics.

The compactification we construct here can be used to compute characteristic numbers for families of singular cubics as well; generally speaking, the difficulty of the task increases with the codimension of the family, in contrast with the degeneration method.

We give a sequence of 5 blow-ups $V_{1}, \ldots, V_{5}$ over $\mathbb{P}^{9}=V_{0}$, with nonsingular centers $B_{i} \hookrightarrow V_{i}$; if $F \subset \mathbb{P}^{9}$ is a family of reduced cubics, and $F_{1}, \ldots F_{5}$ are the proper transforms in the blow-ups of its closure $F_{0}$ in $\mathbb{P}^{9}$, we basically translate the problem of computing the characteristic numbers for $F$ into one of computing the five Segre classes $s\left(B_{i} \cap F_{i}, F_{i}\right), i=0, \ldots, 4$. Now, in general these are easier to compute when the codimension of $F$ is low; for $F$ the family of smooth cubics, $F_{i}=V_{i}$ and $s\left(B_{i}, V_{i}\right)$ are the inverse Chern classes of the normal bundles to $B_{i}$ in $V_{i}$, which are obtained in the blow-up construction. The classes needed when $F$ parametrizes other families (e.g. nodal cubics, or cuspidal cubics, or cubics tangent to a line at a given point) will require some more work.

In the $\mathbb{P}^{9}$ parametrizing plane cubic curves, call 'point-conditions' and 'lineconditions' respectively the hypersurfaces consisting of the cubics respectively containing a given point and tangent to a given line. The intersection of all line-conditions in $\mathbb{P}^{9}$ is supported on a four-dimensional irreducible variety $S$ parametrizing all nonreduced cubics-i.e., cubics decomposing into a line and a 'double line'. For any family of reduced cubics $F \subset \mathbb{P}^{9}-S$, consider the number $N$ of elements (counted with multiplicity) in the intersection of $F$ with given general point- and line-conditions. For example, if $F$ is the set of all smooth cubics, $N$ is a characteristic number for smooth cubics.

Now, for any variety mapping to $\mathbb{P}^{9}$, isomorphically over $\mathbb{P}^{9}-S$, call 'point-' and 'line-conditions' the proper transforms of the conditions in $\mathbb{P}^{9}$; we say that such a variety $\widetilde{V}$ is a 'variety of complete plane cubics' if the intersection of its line-conditions is empty. In $\S 1$ we prove (Theorem I) that the number $N$ is 
precisely the degree of the intersection of the point- and line-conditions in such a $\widetilde{V}$ with the proper transform $\widetilde{F} \subset \widetilde{V}$ of the closure of $F$.

In $\S 3$ we construct a smooth variety $\widetilde{V}$ of complete cubics. This is obtained by a sequence of five blow-ups along nonsingular centers, starting with the blowup of the $\mathbb{P}^{9}$ of cubics along the Veronese of 'triple lines'. The same sequence was considered by U. Sterz, who also obtains some enumerative results (see in particular [St, IV]), and to which we address the reader for a different point of view. The general aim is to separate the proper transforms of the lineconditions above $S$; we accomplish this by systematically blowing up the largest component of their intersection. In doing so, we also collect (Theorem III) the information required to compute in $\widetilde{V}$ the intersection degrees we need: i.e., a description of the intersection rings of the centers of the blow-ups, the total Chern classes of their normal bundles, and information consisting essentially of the multiplicities of the conditions along the centers.

The computation of the intersection degrees is performed by using a formula (Theorem II in §2) which relates intersections under blow-ups. For $X_{\nu}$ subschemes of a scheme $V$, and $\tilde{V}$ the blow-up of $V$ along a regularly imbedded subscheme $B$, the formula gives the difference between the intersection number of the $X_{\nu}$ in $V$ and the intersection number of their proper transforms in $\widetilde{V}$ explicitly, in terms of information essentially equivalent to the Segre classes $s\left(B \cap X_{\nu}, X_{\nu}\right)$. We can apply this formula to climb the sequence of blow-ups defining our variety of complete cubics.

In view of Theorems I, II, and III, the key information for computing the characteristic numbers for any family $F$ of cubics amounts to five Segre classes $s\left(B_{i} \cap F_{i}, F_{i}\right)$, where $F_{i}$ are the proper transforms of the closure of $F$ in $\mathbb{P}^{9}$. In fact, this result is best expressed in terms of equivalent data, i.e. the "full intersection classes'

$$
B_{i} \circ F_{i}=c\left(N_{B_{i}} V_{i}\right) \cap s\left(B_{i} \cap F_{i}, F_{i}\right) .
$$

Theorem IV gives the numbers for a family $F$ of reduced cubics explicitly in terms of the classes $B_{i} \circ F_{i}$. For the family $F$ of all smooth cubics we have $F_{i}=V_{i}$, thus $B_{i} \circ F_{i}=B_{i}$ (since, for $B, V$ smooth, the Segre class $s(B, V)$ equals the inverse total Chern class $\left.c\left(N_{B} V\right)^{-1}\right)$. This allows us to get the characteristic numbers for smooth cubics by simply evaluating coefficients of certain power series (Corollary IV).

For $F$ the family of nodal cubics, or of cuspidal cubics, etc., the computation of the classes $B_{i} \circ F_{i}$ is a more challenging task. We will devote to it a second note.

A good example of a less trivial application of Theorem IV to smooth cubics is the computation of the characteristic numbers obtained by considering also the codimension- 2 condition expressing the tangency to a line at a given point. To apply Theorem IV to this question, we have to compute the five classes for the family of cubics satisfying one of these conditions. This computation is 
sketched in $\S 5$; the characteristic numbers (agreeing with Maillard and Zeuthen's results) are listed in Corollary IV ${ }^{\prime}$. In fact, we show that the information we need to compute the numbers with respect to codimension-1 conditions for any family of cubics (i.e. the five classes) is enough to obtain the results involving these codimension- 2 conditions as well (Theorem IV ${ }^{\prime}$ in $\S 5$ ). This result will also be applied to families of singular cubics in the future note.

In this paper we work over an algebraically closed field of characteristic $\neq$ 2,3 . The blow-up formula in $\S 2$ is characteristic-free, and the preliminary results (in particular Corollary I) hold in characteristic $\neq 2$; however, the blowup construction for the space of cubics needs characteristic $\neq 2,3$.

Some of the material in this paper appears in the author's doctoral thesis written under the guidance of W. Fulton at Brown (May 1987), and (in a sketchier version) in [A].

Aknowledgements. It is a pleasure to thank A. Collino and W. Fulton for proposing the problem and for constant advice and encouragement. I also want to thank Joe Harris for several enlightening comments on the subject.

\section{Preliminaries: varieties of Complete Plane curves}

We will discuss here some facts and notations we will use in the rest of the note. The facts hold for any degree and any family of reduced curves, so we will not restrict ourselves to smooth cubics.

In the $\mathbb{P}^{N}$ parametrizing plane curves of degree $d$, call point-conditions and line-conditions respectively the hypersurfaces consisting of the plane curves respectively containing a given point and tangent to a given line. By 'tangent to a line' we will always mean 'intersecting a line with multiplicity at least 2 at a point'. We will say that a curve $c$ and a line $l$ are 'properly tangent' if $l$ is simply tangent to $c$ at a single nonsingular point-i.e., if the tangency point is smooth on $c$ and $c$ is a smooth point of the line-condition corresponding to $l$.

For any variety $\widetilde{V}$ mapping birationally to $\mathbb{P}^{N}$, biregularly over the set $\mathbb{P}^{N}-$ $S$ consisting of reduced curves, call the proper transforms of the point- and the line-conditions of $\mathbb{P}^{N}$ point- and line-conditions of $\widetilde{V}$.

Definition. We shall say that $\widetilde{V}$ is a variety of complete plane curves of degree $d$ if, moreover, the intersection of all its line-conditions is empty.

The general point- and line-conditions of $\widetilde{V}$ define divisors $\widetilde{P}, \widetilde{L}$ in $\widetilde{V}$. Although in general these need not be Cartier divisors on $\widetilde{V}$, notice that they restrict to Cartier divisors on the inverse image of $\mathbb{P}^{N}-S$ : thus if their intersection with a subvariety $\widetilde{F}$ of $\widetilde{V}$ is proper and does not have components lying over $S$, then intersection products $\widetilde{P} \cdot \widetilde{F}$ and $\widetilde{L} \cdot \widetilde{F}$ are defined. When writing such products, we will imply that this is the case. 
Our aim in this section is to show

Theorem I. Let $\tilde{V}$ be a variety of complete plane curves of degree $d, F$ an $r$-dimensional (maybe noncomplete) subvariety in $\mathbb{P}^{N}$ parametrizing a family of reduced curves, and let $\widetilde{F}$ be the proper transform in $\widetilde{V}$ of the closure of $F$. Then the number of elements (counted with multiplicities) of $F$ containing $n_{p}$ given points and tangent to $n_{l}$ given lines in general position, with $n_{p}+n_{l}=r$, is $\widetilde{P}^{n_{p}} \cdot \widetilde{L}^{n_{l}} \cdot \widetilde{F}$. Furthermore, the elements containing the given points and properly tangent to the given lines are counted with multiplicity 1.

Note that the statement implies that this number does not change when $F$ is replaced with any dense open subset of $F$. I.e., 'special' curves in the family can be discarded.

In this note, our main application of this result is to the computation of the characteristic numbers for the family of smooth plane cubics. Since in characteristic $\neq 2$ the general smooth curve is reflexive (so that for general lines all tangencies will be proper), Theorem I gives

Corollary I. The characteristic numbers for the family of smooth plane curves of degree $d$ are given by $\widetilde{P}^{n_{p}} \cdot \widetilde{L}^{n_{l}}$, for all $n_{p}, n_{l}$ with $n_{p}+n_{l}=\frac{d(d+3)}{2}$.

In $\S 3$ we will construct a 'variety of complete plane cubics'; Corollary I will then allow us to explicitly perform the computation for smooth plane cubics. More generally, Theorem I and the construction in $\S 3$ will give a tool (Theorem IV in $\S 4$ ) to compute the numbers for any family of reduced cubics, on the basis of geometric information.

Let $Q$ be a 3-dimensional vector space over an algebraically closed field of characteristic $\neq 2$. The curves of degree $d$ in the projective plane $\mathbb{P}^{2}=\mathbf{P}(Q)$ form a projective space $\mathbb{P}^{N}=\mathbb{P}\left(\operatorname{Sym}^{d} \check{Q}\right)$, of dimension $N=\frac{d(d+3)}{2}$. In this projective space, the curves that contain a given point form a hyperplane; while those that are tangent to a given line form a hypersurface of degree $2 d-2$. We will call these divisors point-conditions and line-conditions respectively. As a point varies in $\mathbb{P}^{2}$, the corresponding point-condition traces a subset of $\check{\mathbb{P}}^{N}$. In fact, if $\left(x_{0}: x_{1}: x_{2}\right)$ are coordinates in $\mathbb{P}^{2}$, the point-condition corresponding to the point $\left(\bar{x}_{0}: \bar{x}_{1}: \bar{x}_{2}\right)$ is the hyperplane in $\mathbb{P}^{N}$ whose equation has the monomials of degree $d$ in $\bar{x}_{0}, \bar{x}_{1}, \bar{x}_{2}$ for coefficients. In other words:

Remark 1. The set of point-conditions of $\mathbb{P}^{N}$ is the $d$-Veronese imbedding of $\check{\mathbb{P}}^{2}$ in $\check{\mathbb{P}}^{N}$. In particular, the set of point-conditions is nondegenerate and irreducible, and in particular it is not contained in any finite union of hyperplanes: for example, it follows that a point-condition can always be chosen to cut properly finitely many arbitrary subvarieties of $\mathbb{P}^{N}$.

On the open subset of $\mathbb{P}^{N}$ formed by the smooth curves, an injective morphism is defined to the space $\mathbb{P}^{M}=\mathbb{P}\left(\operatorname{Sym}^{d(d-1)} \check{Q}\right)$ parametrizing degree$d(d-1)$ curves, by associating to each curve its dual. Note that a curve is 
tangent to a line $l \subset \mathbb{P}^{2}$ if and only if its dual contains $l \in \check{\mathbb{P}}^{2}$ : thus the line-conditions in $\mathbb{P}^{N}$ are the pull-backs of the point-conditions in the $\mathbb{P}^{M}$ parametrizing all degree- $d(d-1)$ plane curves, and it follows (by Remark 1)

Remark 2. The set of line-conditions of $\mathbb{P}^{N}$ is the $d(d-1)$ th Veronese imbedding of $\check{\mathbb{P}}^{2}$ into $\check{\mathbb{P}}^{M}$.

This also makes it clear that the rational map $\psi: \mathbb{P}^{N} \rightarrow \mathbb{P}^{M}$ determined by the morphism above is defined by the linear system generated by the lineconditions in $\mathbb{P}^{N}$.

We want to resolve the indeterminacies of $\psi$. These occur on the intersection of all line-conditions, supported on the variety $S \subset \mathbb{P}^{N}$ parametrizing nonreduced curves; $\psi$ is an injective morphism on $\mathbb{P}^{N}-S$. We will call any variety $\widetilde{V}$ filling the commutative diagram

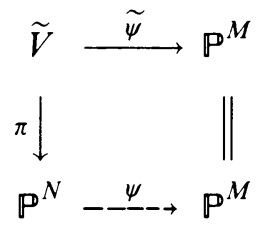

with $\tilde{\psi}$ a morphism, and isomorphic to $\mathbb{P}^{N}$ outside $\pi^{-1}(S)$, a variety of complete plane curves of degree $d$ '. For example, the blow-up of $\mathbb{P}^{N}$ along the scheme-intersection of all its line-conditions is a variety of complete curves of degree $d$. An instance is the classical 'variety of complete conics' (cf. [CX, §2]). In a different contest, Vainsencher's varieties of complete quadrics (inspired by Schubert's work) give another example of a similar situation.

Note that the first condition ( $\psi$ lifting to a morphism $\widetilde{V} \rightarrow \mathbb{P}^{M}$ ) amounts to just requiring that the intersection in $\widetilde{V}$ of the proper transforms of all lineconditions be empty. We will construct a smooth variety of complete cubics by blowing-up $\mathbb{P}^{9}$ five times along suitable centers, and use this variety to compute the characteristic numbers of certain families of plane cubics.

The proper transforms of general point-conditions and line-conditions determine classes $\widetilde{P}, \widetilde{L}$ of divisors on $\widetilde{V}$. If $\widetilde{V}$ is smooth, then for any $\widetilde{F} \subset \widetilde{V}$ we have intersection products $\widetilde{P} \cdot \widetilde{F}$ and $\widetilde{L} \cdot \widetilde{F}$. Even if $\widetilde{V}$ is not smooth, however, $\widetilde{P}$ and $\widetilde{L}$ restrict to Cartier divisors on $\pi^{-1}\left(\mathbb{P}^{N}-S\right.$ ) (since this is smooth): thus $\widetilde{P} \cdot \widetilde{F}, \widetilde{L} \cdot \widetilde{F}$ are defined as long as $\pi(F) \nsubseteq S$ and the proper transforms of general choices of conditions cut $\widetilde{F} \cap \pi^{-1} S$ properly. This will always be the case for $F$ as below (see Proposition 1 (1) and Lemma 1).

Computing the characteristic numbers for families of reduced plane curves amounts to computing the number of intersections of certain subsets of $\mathbb{P}^{N}$ with assortments of point- and line-conditions in general position.

Let us first consider line-conditions. Let $F$ be a pure $r$-dimensional locally closed (maybe noncompact) subset of $\mathbb{P}^{N}$, parametrizing a family of reduced curves: i.e., we assume $F \cap S=\varnothing$. For example, $F$ could be the set of all 
smooth curves, or the set of all nodal curves, or the set of all nodal curves containing a given point, and so on. The number of elements of $F$ tangent to $r$ lines in general position in the plane is the number of points of intersection of $F$ with $r$ general line-conditions of $\mathbb{P}^{M}$; but since all line-conditions contain the set of nonreduced curves, often nonreduced curves will appear in the intersection of $r$ (general) line-conditions with the closure $\bar{F}$ of $F$. For example, if $F$ is the set of nonsingular conics, the intersection of 5 general line-conditions with $\bar{F}$ (= the whole of $\mathbb{P}^{5}$ ) consists of one isolated point and of the 2-dimensional set of 'double lines'.

Let then $\widetilde{V}$ be a variety of complete plane curves of degree $d, \widetilde{F}$ the proper transform of $\bar{F}$ in $\widetilde{V}$, and call 'line-conditions in $\widetilde{V}$ ' the proper transforms in $\widetilde{V}$ of the line-conditions of $\mathbb{P}^{N}$; call $\widetilde{L}$ the class of the general line-condition in $\widetilde{V}$.

Proposition 1. A line-condition in $\widetilde{V}$ can always be chosen to cut properly any finite collection of subvarieties of $\widetilde{V}$;

With $F$ as above, $r$ line-conditions in $\widetilde{V}$ can be chosen to cut $\widetilde{F}$ in finitely many points, mapping to points of $F$; the number of elements of $F$ that are tangent to $r$ lines in general position is the number of intersections of $\widetilde{F}$ with $r$ general line-conditions in $\widetilde{V}$.

Proof. (1) follows from Remark 2: the set of line-conditions is not contained in any finite union of hyperplanes of $\check{\mathrm{P}}^{M}$.

For (2), let $\pi_{F}$ be the restriction of $\pi$ to $\widetilde{F}$, and set $E=\overline{\widetilde{F}-\pi_{F}^{-1} F}$ : $\operatorname{dim} E \leq r-1$, so (2) follows by applying (1) $r$ times.

(3) follows from (2).

Working in a variety of complete curves $\widetilde{V}$, the number we are after is the number of points of intersections of complete subsets of $\widetilde{V}$ : counting multiplicities, the number is given by the degree of $\widetilde{L}^{r} \cdot \widetilde{F}$.

Now for the point-conditions. As above, let $\widetilde{V}$ be a variety of complete plane curves of degree $d$, mapping to $\mathbb{P}^{N}$ by $\pi, F$ a locally closed subset of $\mathbb{P}^{N}, r=\operatorname{dim} F$ and $\widetilde{F}$ the proper transform in $\widetilde{V}$ of the closure $\bar{F}$ of $F$. In general, $\sim$ denotes proper transform via $\pi$.

Lemma 1. There exists a point-condition $P$ such that $\overline{P \cap F}=P \cap \bar{F}, \widetilde{P \cap \bar{F}}=$ $\widetilde{P} \cap \widetilde{F}$, and $\operatorname{dim}(\widetilde{P} \cap \widetilde{F})=r-1$.

Proof. Let $\pi_{F}$ be the restriction of $\pi$ to $\widetilde{F} . \overline{P \cap F}=P \cap \bar{F}$ and $\operatorname{dim}(\widetilde{P} \cap \widetilde{F})=$ $r-1$ are forced by requiring that $P$ cut properly $F$ and $\bar{F}-F$. Next, certainly $\widetilde{P \cap \bar{F}}$ coincides with $\widetilde{P} \cap \widetilde{F}$ outside $\pi_{F}^{-1}(S)$ for any point-condition $P$; we have to show that we can choose $P$ so that none of the components of $\widetilde{P} \cap \widetilde{F}$ lies in $\pi_{F}^{-1}(S)$. Let then $F_{i} \subset \bar{F}$ be the supports of the components of $\pi_{F}^{-1}(S)$, and choose the point-condition $P$ so that it cuts properly all the $F_{i}$ 's. Since $\pi_{F}^{-1}(S)$ itself has dimension (at most) $r-1$, this will force $\operatorname{dim} \pi_{F}^{-1}(S) \cap \widetilde{P}<r-1$, and we will be done. That a point-condition can be chosen to cut properly any finite 
choice of subvarieties of $\mathbb{P}^{N}$ is once more a consequence of the nondegeneracy of the set of point-conditions (Remark 1).

Following our line of notations, call now point-conditions in $\widetilde{V}$ the proper transforms in $\widetilde{V}$ of the point-conditions in $\mathbb{P}^{N}$; the general ones determine a divisor class $\widetilde{P}$ of $\widetilde{V}$.

From Proposition 1 and Lemma 1, the first part of our basic tool follows:

Theorem I. (1) Let $\widetilde{V}$ be a variety of complete plane curves of degree $d, F$ an r-dimensional subvariety of $\mathbb{P}^{N}$ parametrizing a family of reduced curves, and let $\widetilde{F}$ be the proper transform of $\bar{F}$ in $\widetilde{V}$. Then the number of elements (counted with multiplicities) of $F$ containing $n_{p}$ points and tangent to $n_{l}$ lines in general position, with $n_{p}+n_{l}=r$, is given by $\widetilde{P}^{n_{p}} \cdot \widetilde{L}^{n_{l}} \cdot \widetilde{F}$.

Proof. By repeated applications of Lemma $1, n_{p}$ point-conditions $P_{1}, \ldots, P_{n_{p}}$ can be chosen so that $\left[\overline{\left(\bigcap_{i} P_{i}\right) \cap F}\right]^{\sim}=\left[\left(\bigcap_{i} P_{i}\right) \cap \bar{F}\right]^{\sim}=\left(\bigcap_{i} \widetilde{P}_{i}\right) \cap \widetilde{F}$. To conclude, it suffices to apply Proposition 1 to $\left(\bigcap_{i} P_{i}\right) \cap F$.

The last part of Theorem I concerns intersection multiplicities. It can be proven by induction on $n_{l}$; the start and the induction step are consequences of:

Lemma 2. Let $C$ be an irreducible curve in $\mathbb{P}^{N}$, such that $C \cap S=\varnothing$ and that the curves in $C$ do not have a common component. Let $c$ be a general point of $C$; then

(1) there exist at most finitely many points $p \in \mathbb{P}^{2}$ such that $p \in c$ and the point-condition corresponding to $p$ is tangent to $C$ at $c$;

(2) there exist at most finitely many lines $l \subset \mathbb{P}^{2}$ such that $c$ is properly tangent to $l$ and the line-condition corresponding to $l$ is tangent to $C$ at $c$.

Proof. We can assume $c$ is a smooth point of $C$; if $c$ is reducible as a plane curve, we can in fact assume that all components of $c$ are moving smoothly as $c$ moves on $C$.

(1) By definition, the point-condition $P$ corresponding to $p \in \mathbb{P}^{2}$ contains $c$ if and only if $p \in c . P$ is tangent to $C$ at $c$ if it contains the tangent line to $C$ at $c$ : let $c^{\prime} \neq c$ be a point of this line. $P$ contains the line through $c$ and $c^{\prime} \neq c$ if and only if $p \in c \cap c^{\prime}$ : since all components of $c$ are moving smoothly, this intersection is finite.

(2) Since $c$ and $l$ are properly tangent, then $c$ is a smooth point of the line-condition $L_{l}$ corresponding to $l$ : therefore, $C$ is tangent to $L_{l}$ at $c$ if and only if $\psi(C)$ is tangent at $\psi(c)$ to the point-condition corresponding to $l$ (since $C \cap S=\varnothing, \psi(c)$ is defined for all $c \in C$ ). Also, $l$ belongs to a reduced component of $\psi(c)$, since the tangency point is smooth on $c$. Since $c$ is general in $C$, we can assume this component is moving smoothly at $\psi(c)$. (2) is then simply the dual of (1): i.e., (1) applied to $\psi(c) \in \psi(C) \subset \mathbb{P}^{M}$. 
Theorem I. (2) In the same hypotheses of Theorem I (1), the elements containing the given points and properly tangent to the given lines appear with multiplicity 1 .

Proof. We can assume that the curves in $F$ do not have a common component: if they do, factoring it out reduces the statement to the same for lower degree curves.

We will prove that: (a) the statement is true for $n_{p}=r, n_{l}=0$; (b) the statement for $n_{p}=r-k, n_{l}=k, k<r$ implies the statement for $n_{p}=$ $r-k-1, n_{l}=k+1$. The assertion will then follow by induction.

(a) It is enough to show that there exists a point-condition $P$ such that $P \cap F$ is reduced, and to apply this fact $r$ times. Now, suppose that is not the case: i.e., suppose that for each point-condition $P, P \cap F$ has some nonreduced component, of dimension $r-1$. These components would cover a component of $F$, and the set of point-conditions is 2-dimensional: thus for a general point $c$ in a component of $F$ there would be infinitely many point-conditions tangent to $F$ at $c$. In particular, they would all be tangent to some curve through $c$, contradicting Lemma 2 (1).

(b) For $n_{p}=r-k, n_{l}=k$, the statement says that for general lineconditions $L_{1}, \ldots, L_{k}$ and point-conditions $P_{1}, \ldots, P_{r-k}$, the intersection $F \cap L_{1} \cap \cdots \cap L_{k} \cap P_{1} \cap \cdots \cap P_{r-k}$ is transversal at all points corresponding to proper tangency to the lines. Consequently, the components $C_{i}$ of the curve $F \cap L_{1} \cap \cdots \cap L_{k} \cap P_{1} \cap \cdots \cap P_{r-k-1}$ that contain these points are reduced and cut transversally by $P_{r-k}$ : to prove the induction step, we must show that there exists a line $l$ in the plane, such that the corresponding line-condition $L_{k+1}$ cuts the $C_{i}$ transversally at points corresponding to proper tangency to $l$. By Lemma 2 (2), the set of line-conditions which fail to cut transversally the $C_{i}$ 's at points corresponding to proper tangencies is at most 1-dimensional, in the 2-dimensional set of line-conditions. Therefore, an $l$ as above must exist.

\section{Preliminaries: AN INTERSECTION FORMULA}

In $\S 3$ we will construct a 'variety of complete plane cubics' $\widetilde{V}$ by a stack of blow-ups at nonsingular centers over $\mathbb{P}^{9}$. Corollary I in $\S 1$ expresses the characteristic numbers as degrees of intersection of the proper transforms in $\widetilde{V}$ of suitable hypersurfaces of $\mathbb{P}^{9}$; we introduce here the formula we will use in $\S 4$ to compute these intersection degrees.

Let $V$ be a nonsingular variety of dimension $n$ over an arbitrary field, and $B$ a nonsingular closed subvariety of codimension $d$ in $V$. For $X \hookrightarrow V$ any pure-dimensional subscheme of $V$, we set

$$
B \circ X=c\left(N_{B} V\right) \cap s(B \cap X, X)
$$

in the Chow group $A_{*}(B \cap X)$ of $B \cap X$. We call this the 'full intersection class' of $X$ by $B$ in $V$. 
Lemma. Denote by $\{\cdot\}_{r}$ the $r$-dimensional component of the class between braces.

(1) Let $N$ be the pull-back of $N_{B} V$ to $B \cap X, C=C_{B \cap X} X \hookrightarrow N$ the cone of $B \cap X$ in $X, \mathscr{Q}$ the universal quotient bundle of rank $d$ of $\mathbb{P}(N \oplus 1)$, $p$ the projection $\mathbb{P}(N \oplus 1) \rightarrow B \cap X$. Then

$$
B \circ X=p_{*}(c(\mathscr{Q}) \cap[\mathbb{P}(C \oplus 1)]) ;
$$

(2) $\{B \circ X\}_{\operatorname{dim} X-d}=B \cdot X=j^{\prime}[X]$;

(3) $\{B \circ X\}_{\nu}=0$ for $\nu<\operatorname{dim} X-d, \nu>\operatorname{dim} B \cap X$.

Proof. (1) Denote by $\mathscr{O}(-1)$ the universal line bundle on $\mathbb{P}(N \oplus 1)$; then $\mathscr{Q}=p^{*} N \oplus 1 / \mathscr{O}(-1)$, and therefore

$$
\begin{aligned}
p_{*}(c(\mathscr{Q}) \cap[\mathbb{P}(C \oplus 1)]) & =p_{*}\left(c\left(p^{*} N\right) \cap\left(1-c_{1}(\mathscr{O}(1))^{-1} \cap[\mathbb{P}(C \oplus 1)]\right)\right) \\
& =p_{*}\left(c\left(p^{*} N\right) \cap\left(\sum_{i \geq 0} c_{1}(\mathscr{O}(1))^{i} \cap[\mathbb{P}(C \oplus 1)]\right)\right) \\
& =c(N) \cap s(C) \\
& =g^{*} c\left(N_{B} V\right) \cap s(B \cap X, X) .
\end{aligned}
$$

(2) See [F, Proposition 6.1(a)] and $\S 6.2$ ( $j$ ! is the 'Gysin homomorphism').

(3) $\{B \circ X\}_{\nu}=0$ for $\nu>\operatorname{dim} B \cap X$ is obvious; $\{B \circ X\}_{\nu}=0$ for $\nu<$ $\operatorname{dim} X-d$ follows from (1).

Let $\widetilde{V}$ be the blow-up of $V$ along $B$, suppose $X_{1}, \ldots, X_{r}$ are pure-dimensional subschemes of $V$, and let $\widetilde{X}_{\nu} \subset \widetilde{V}$ be their proper transforms: i.e., the blow-ups of $X_{\nu}$ along $B \cap X_{\nu}$.

Theorem II. Suppose that the codimensions of the $X_{\nu}$ add to the dimension of $V$, and that the intersection $\cap X_{\nu}$ is a proper scheme. With the notation above

$$
\int_{\widetilde{V}} \tilde{X}_{1} \cdots \widetilde{X}_{r}=\int_{V} X_{1} \cdots X_{r}-\int_{B} \frac{\prod_{\nu=1}^{r}\left(B \circ X_{\nu}\right)}{c\left(N_{B} V\right)} .
$$

Here the first product is taken in $\widetilde{V}$, the second in $V$ and the third in $B$. In $\S 4$ this formula will be applied to each blow-up in the sequence.

Proof. Let $E=\mathbb{P}\left(N_{B} V\right)$ be the exceptional divisor of the blow-up, and write the maps involved as follows:

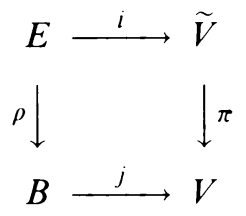


Theorem II follows from

Claim. Let $X_{1}, \ldots, X_{r}$ be pure-dimensional subschemes of $V$, and write $m=$ $\operatorname{dim} V-\operatorname{codim}_{V} X_{1}-\cdots-\operatorname{codim}_{V} X_{r}$. Then

$$
\pi^{*}\left(X_{1} \cdots X_{r}\right)=\tilde{X}_{1} \cdots \tilde{X}_{r}+i_{*}\left\{\frac{\rho^{*}\left(\left(B \circ X_{1}\right) \cdots\left(B \circ X_{r}\right)\right)}{c\left(N_{E} \tilde{V}\right)}\right\}_{m}
$$

in $A_{m}\left(\bigcap_{i} \pi^{-1} X_{i}\right)$.

By [F, Proposition $6.7(\mathrm{~d})]$, to prove $(*)$ one must show that the equality holds after (1) pushing it forward on $V$ by $\pi$, and (2) pulling it back on $E$ by $i$. We will show (1) here (which is enough to imply Theorem II), and leave (2) to the interested reader.

By the the projection formula, (1) amounts to

$$
X_{1} \cdots X_{r}=\pi_{*}\left(\tilde{X}_{1} \cdots \tilde{X}_{r}\right)+j_{*}\left\{\frac{\prod_{\nu=1}^{r}\left(B \circ X_{\nu}\right)}{c\left(N_{B} V\right)}\right\}_{m} .
$$

Example 12.4.4 in $[\mathrm{F}]$ gives

$$
X_{1} \cdots X_{r}=\pi_{*}\left(\tilde{X}_{1} \cdots \tilde{X}_{r}\right)+j_{*} p_{*}\left(\mathbb{P}\left(C_{1} \oplus 1\right) \cdots \mathbb{P}\left(C_{r} \oplus 1\right)\right),
$$

where $p$ denotes the projection $\mathbb{P}\left(N_{B} V \oplus 1\right) \rightarrow B$ and $C_{i}=C_{B \cap X_{i}} X_{i}$ are the normal cones of the imbeddings $B \cap X_{i} \hookrightarrow X_{i}$. Thus to prove (1) we may show that

$$
p_{*}\left(\mathbb{P}\left(C_{1} \oplus 1\right) \cdots \cdot \mathbb{P}\left(C_{r} \oplus 1\right)\right)=\left\{\frac{\left(B \circ X_{1}\right) \cdots\left(B \circ X_{r}\right)}{c\left(N_{B} V\right)}\right\}_{m},
$$

where $p$ is the projection $\mathrm{P}(N \oplus 1) \rightarrow B$.

To this effect, let $d=\operatorname{codim}_{V} B, \zeta=c_{1}\left(\mathscr{O}_{\mathbf{P}(N \oplus 1)}(1)\right)$, and $\mathscr{Q}$ be the universal quotient bundle of rank $d$ over $\mathbb{P}(N \oplus 1)$. Any element $A$ in $A_{k}(\mathbb{P}(N \oplus 1))$ can be expressed uniquely in the form

$$
A=\sum_{\nu=0}^{d} \zeta^{\nu} \cap p^{*} \alpha_{k-d+\nu}
$$

where $\alpha_{j} \in A_{j}(B)$. Setting $\alpha=\bigoplus_{\nu=0}^{d} \alpha_{k-d+\nu} \in A_{*}(B)$, we say that $A$ corresponds to $\alpha$.

Claim 1. $\left[\mathbb{P}\left(C_{i} \oplus 1\right)\right]$ corresponds to $B \circ X_{i}, i=1, \ldots, r$.

Indeed, for any $\alpha_{j} \in A_{j} B$ and any $\nu \leq d$, by Example 3.3.3 in [F]

$$
p_{*}\left(c(\mathscr{Q}) \cap \zeta^{\nu} \cap p^{*} \alpha_{j}\right)=\alpha_{j}
$$

thus the $\left[\mathbb{P}\left(C_{i} \oplus 1\right)\right]$ must correspond to $p_{*}\left(c(\mathscr{Q}) \cap\left[\mathbb{P}\left(C_{i} \oplus 1\right)\right]\right)$. This equals $B \circ X_{i}$ by (1) of the lemma.

Next, we relate in the above terminology intersections in $\mathbb{P}(N \oplus 1)$ and $B$. With $n=\operatorname{dim} V=\operatorname{dim} \mathbb{P}(N \oplus 1)$ : 
Claim 2. Suppose $A_{i} \in A_{k_{i}}(\mathbb{P}(N \oplus 1))$ correspond to $\alpha^{(i)}, i=1, \ldots, r$, and let $m=k_{1}+\cdots+k_{r}-(r-1) n$. Then

$$
p_{*}\left(A_{1} \cdots A_{r}\right)=\left\{\frac{\alpha^{(1)} \cdots \alpha^{(r)}}{c(N)}\right\}_{m} .
$$

Indeed, by linearity we may assume $A_{i}=\zeta^{q_{i}} \cap p^{*} \alpha^{(i)}$, with $\alpha^{(i)} \in A_{k_{i}-d+q_{i}} B$. Setting $q=\sum q_{i}$ and applying the projection formula, the claim reduces to

$$
p_{*}\left(\zeta^{q} \cap p^{*} \alpha^{(r)}\right)=s_{q-d}(N) \cap \alpha^{(r)},
$$

which amounts to the definition of $s(N)=c(N)^{-1}[\mathrm{~F}, \S 3.1]$.

Claims 1 and 2 give $\left(1^{\prime}\right)$, concluding the proof of (1).

We remark that $(*)$ above (and therefore Theorem II) holds for possibly singular $V$ and $B$, if $B$ is regularly imbedded in $V$ and under conditions that guarantee the intersection products are defined.

One advantage in writing the formula in Theorem II in terms of full intersection classes is that these are often easy to express 'concretely'. In particular:

(i) if $X_{\nu}=V$, then $B \circ X_{\nu}=[B]$. Indeed, in this case $s\left(B \cap X_{i}, X_{i}\right)=s(B, V)$ is the inverse total Chern class $c\left(N_{B} V\right)^{-1}$.

(ii) If $X_{\nu}$ is a divisor then $B \circ X_{\nu}=e_{B} X_{\nu}[B]+j^{*}\left[X_{\nu}\right]$, where $e_{B} X$ denotes the multiplicity of $X$ along $B$ and $j$ is the imbedding $B \hookrightarrow V$.

(iii) Similarly, if $X_{\nu}$ has codimension 2 and meets $B$ in an irreducible $W$, with $\operatorname{dim} W=\operatorname{dim} B-1$, then $B \circ X_{\nu}=e_{W} X_{\nu}[W]+j^{*}\left[X_{\nu}\right]$.

(These statement follow from (2), (3) in the lemma.)

By use of Theorem II, the characteristic numbers for a family $F \subset \mathbb{P}^{9}$ will be expressed in terms of certain full intersection classes related to $F$ (Theorem IV, §4). For $F$ the family of smooth cubics, we will just have to apply (i). To build up Theorem IV, we will need to compute full intersection classes related to point- and line- conditions, using (ii) (see §3); and (iii) will be needed for further computations in $\S 5$.

\section{A SMOOTH VARIETY OF COMPLETE CUBICS}

Assume hereafter that the characteristic of the ground field is $\neq 2,3$. In this section we will construct a smooth variety of complete plane cubics, by means of a stack of blow-ups over $\mathbb{P}^{9}$. The same sequence of blow-ups was obtained independently by U. Sterz (cf. [St]); he gives a detailed description in coordinates of each of them, and computes their homology bases and several relations. Our point of view differs from Sterz's in the sense that we need to obtain 'geometric' information regarding the blow-ups, to apply the intersection formula of $\S 2$. More precisely, we need for each blow-up $V_{i+1}=\mathrm{B} l_{B_{i}} V_{i}$ a description of the intersection ring of each center $B_{i}$ and the total chern class $c\left(N_{B_{i}} V_{i}\right)$ of its normal bundle; also, we need the full intersection classes of the proper transforms of point- and line-conditions in each blow-up with respect to 
the center. The result is

Theorem III. A smooth variety $\tilde{V}=V_{5}$ of complete cubics can be obtained by a sequence of 5 blow-ups $V_{i}=B l_{B_{i-1}} V_{i-1}, i=1, \ldots, 5$, with $V_{0}=\mathbb{P}^{9}$, and where

(1) $B_{0} \cong \mathbb{P}^{2}$ is the locus of 'triple lines' in the space $V_{0}=\mathbb{P}^{9}$ of plane cubics; the intersection ring of $B_{0}$ is generated by the hyperplane class $h$, and $\int h^{2}=1$;

$$
c\left(N_{B_{0}} V_{0}\right)=\frac{(1+3 h)^{10}}{(1+h)^{3}} .
$$

(2) $B_{1}$ is a rank-2 projective bundle over $B_{0}$; the intersection ring of $B_{1}$ is generated by the pull-back $h$ of $h$ and by the class $\varepsilon$ of the universal line bundle, and

$$
\begin{gathered}
\int_{B_{1}} h^{4}=0, \quad \int_{B_{1}} h^{3} \varepsilon=0, \quad \int_{B_{1}} h^{2} \varepsilon^{2}=1 \\
\int_{B_{1}} h \varepsilon^{3}=9, \quad \int_{B_{1}} \varepsilon^{4}=51 ; \\
c\left(N_{B_{1}} V_{1}\right)=(1+\varepsilon) \frac{(1+3 h-\varepsilon)^{10}}{(1+2 h-\varepsilon)^{6}} .
\end{gathered}
$$

(3) $B_{2}$ is a rank-3 projective bundle over $B_{1}$; the intersection ring of $B_{2}$ is generated by the pull-backs $h, \varepsilon$ of $h, \varepsilon$ and by the class $\varphi$ of the universal line bundle, and

$$
\begin{array}{lll}
\int_{B_{2}} \varphi^{7}=-210, & \int_{B_{2}} \varphi^{6} h=-90, & \int_{B_{2}} \varphi^{6} \varepsilon=-240, \\
\int_{B_{2}} \varphi^{5} h^{2}=-10, & \int_{B_{2}} \varphi^{5} h \varepsilon=0, & \int_{B_{2}} \varphi^{5} \varepsilon^{2}=105, \\
\int_{B_{2}} \varphi^{4} h^{2} \varepsilon=4, & \int_{B_{2}} \varphi^{4} h \varepsilon^{2}=18, & \int_{B_{2}} \varphi^{4} \varepsilon^{3}=42, \\
\int_{B_{2}} \varphi^{3} h^{2} \varepsilon^{2}=-1, & \int_{B_{2}} \varphi^{3} h \varepsilon^{3}=-9, & \int_{B_{2}} \varphi^{3} \varepsilon^{4}=-51,
\end{array}
$$

(all other codimension-7 terms have degree 0 );

$$
c\left(N_{B_{2}} V_{2}\right)=(1+\varphi)(1+\varepsilon-\varphi) .
$$

(4) $B_{3}$ is isomorphic to the blow-up $B l_{\Delta} \mathbb{P}^{2} \times \mathbb{P}^{2}$ of $\mathbb{P}^{2} \times \mathbb{P}^{2}$ along the diagonal; the intersection ring of $B_{3}$ is generated by the pull-backs $l, m$ of the hyperplane classes in the factors of $\mathbb{P}^{2} \times \mathbb{P}^{2}$, and by the class $e$ of the exceptional divisor, and $e m=e l, l^{3}=m^{3}=0$,

$$
\begin{array}{ll}
\int_{B_{3}} l^{2} m^{2}=1, & \int_{B_{3}} e^{2} l^{2}=-1, \\
\int_{B_{3}} e^{3} l=-3, & \int_{B_{3}} e^{4}=-6
\end{array}
$$




$$
\begin{aligned}
c\left(N_{B_{3}} V_{3}\right)=1 & +7 l+17 m-16 e+126 m^{2}+99 l m+21 l^{2} \\
& -315 e l+105 e^{2}+582 l m^{2}+237 l^{2} m-2517 e l^{2}+1611 e^{2} l \\
& -358 e^{3}+1026 l^{2} m^{2}+9174 e^{2} l^{2}-3912 e^{3} l+652 e^{4} .
\end{aligned}
$$

(5) $B_{4}$ is isomorphic to $B_{3}$;

the intersection ring of $B_{4}$ is therefore generated by $l, m, e$ as above;

$$
\begin{aligned}
c\left(N_{B_{4}} V_{4}\right)= & 1-5 l+5 m+18 m^{2}-27 l m+3 l^{2}+21 e l-7 e^{2}-30 l m^{2} \\
& +75 l^{2} m-225 e l^{2}+135 e^{2} l-30 e^{3}+75 l^{2} m^{2} .
\end{aligned}
$$

Also, in these notations:

Full intersection classes. The full intersection classes with respect to the $B_{i}$ 's of the proper transforms $P_{i}, L_{i}$ in $V_{i}$ of point- and line-conditions are

$$
\begin{array}{ll}
B_{0} \circ P_{0}=3 h, & B_{0} \circ L_{0}=2+12 h, \\
B_{1} \circ P_{1}=3 h, & B_{1} \circ L_{1}=1+12 h-2 \varepsilon, \\
B_{2} \circ P_{2}=3 h, & B_{2} \circ L_{2}=1+12 h-2 \varepsilon-\varphi, \\
B_{3} \circ P_{3}=l+2 m, & B_{3} \circ L_{3}=1+4 l+8 m-6 e, \\
B_{4} \circ P_{4}=l+2 m, & B_{4} \circ L_{4}=1+l+5 m-2 e .
\end{array}
$$

The rest of this $\S 3$ is devoted to the proof of Theorem III. Most of the notations employed here appear in the following diagram:

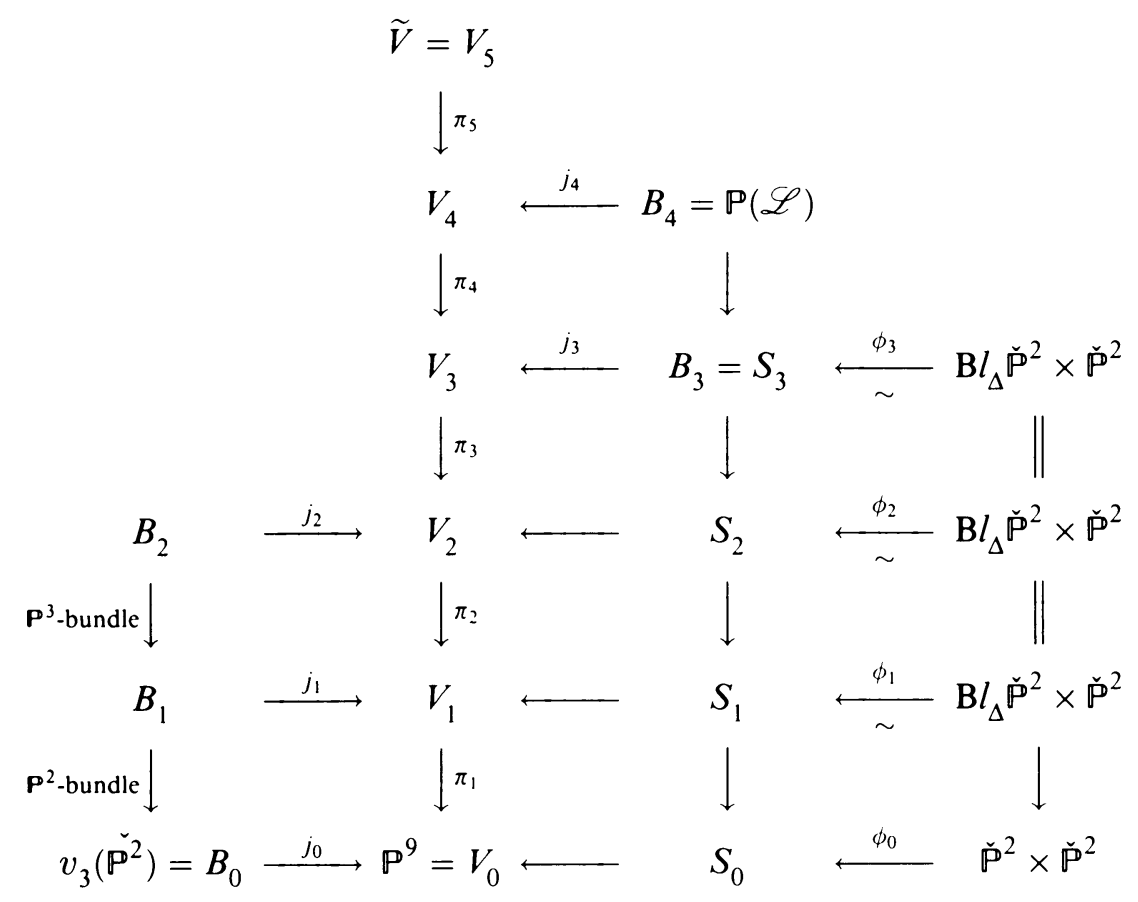


Here $S_{0}$ is the locus of nonreduced cubics, and $B_{0}=v_{3}\left(\check{\mathbb{P}}^{2}\right) \hookrightarrow \mathbb{P}^{9}$ is the Veronese of triple lines. Each $B_{i}$ is the center of the blow-up $V_{i+1}=\mathrm{B} l_{B_{i}} V_{i}$; $S_{i+1}$ denotes the proper transform of $S_{i}$ under this blow-up.

Also, $\mathscr{L}$ is a certain subline bundle of the normal bundle $N_{B_{3}} V_{3}$ of $B_{3}$ in $V_{3} . \Delta$ is the diagonal in $\check{\mathbb{P}}^{2} \times \check{\mathbb{P}}^{2}$.

Finally, $P_{0}, L_{0}$ will respectively be point- and line-conditions in $\mathrm{P}^{9} ; P_{i}$, $L_{i}$ will be the proper transforms of $P_{i-1}, L_{i-1}$, and $E_{i}$ will be the exceptional divisor of the $i$ th blow-up. The $P_{i}$ 's and $L_{i}$ 's will be called 'point-conditions' and 'line-conditions' in $V_{i}$. We will also say that point- and line-conditions are 'in general position' if the corresponding points and lines are.

$\S \S 3.0-3.5$ describe the sequence of blow-ups in some detail. The aim is to 'separate' the line-conditions by blowing-up nonsingular subvarieties; thus, we will generally choose as centers the 'biggest' nonsingular components of the intersection of the line-conditions. In $\mathbb{P}^{9}$ the intersection of the line-condition is supported on the 4-dimensional variety $S_{0}$ parametrizing non-reduced curves. This is the image of a bijective map $\phi_{0}: \check{\mathbb{P}}^{2} \times \check{\mathbb{P}}^{2} \rightarrow \mathbb{P}^{9} ; \phi_{0}$ ramifies along the diagonal, mapping to the 2-dimensional locus $B_{0}$ of 'triple lines'. This latter is our choice for the first blow-up.

Each of $\S \S 3.0-3.5$ is organized as follows: we find on each $V_{i}$, the intersection of all line-conditions, and we choose a center $B_{i}$ for the next blow-up (a nonsingular subvariety or component of the intersection of the line-conditions); then we obtain the information collected in Theorem III. In particular, we describe the intersection rings of the $B_{i}$ 's, and we compute the total Chern class $c\left(N_{B_{i}} V_{i}\right)$ of the normal bundle to $B_{i}$ in $V_{i}$. Next, we examine the geometry of the situation in more detail, to obtain the information we will need in the following stages. Also, we compute the multiplicities $e_{B_{i}} P_{i}, e_{B_{i}} L_{i}$ of the conditions along the centers (in fact, $e_{B_{i}} P_{i}$ will always be 0 ), and the pull-backs $j_{i}^{*} P_{i}, j_{i}^{*} L_{i}$ : this is the information contained in the full intersection classes of the conditions with respect to the centers.

To prove that $V_{5}$ is a 'variety of complete cubics' amounts to proving that the intersection of its line-conditions is empty; this will be shown in §3.5. Equivalently, one can show that $V_{5}$ dominates the graph of the rational map $\psi$ of $\S 1$; a proof in these terms can be found in [St, II, §4].

\subsection{The $\mathbb{P}^{9}$ OF PLANE CUBICS}

Let $Q$ be a 3-dimensional vector space over an algebraically closed field of characteristic $\neq 2,3$, and consider $\mathbb{P}^{9}=\mathbb{P}\left(\operatorname{Sym}^{3} \check{Q}\right)$, the projective space parametrizing cubic curves in the plane $\mathbb{P}^{2}=\mathbb{P} Q . x_{0}, x_{1}, x_{2}\left(\right.$ resp., $\left.a_{0}, \ldots, a_{9}\right)$ will be homogeneous coordinates in $\mathbb{P}^{2}$ (resp., in $\left.\mathbb{P}^{9}\right)$ : the point $\left(a_{0}: \ldots: a_{9}\right) \in$ 
$\mathbb{P}^{9}$ is associated with the cubic of equation

$$
\begin{aligned}
a_{0} x_{0}^{3}+a_{1} x_{0}^{2} x_{1}+a_{2} x_{0}^{2} x_{2}+ & a_{3} x_{0} x_{1}^{2}+a_{4} x_{0} x_{1} x_{2} \\
& +a_{5} x_{0} x_{2}^{2}+a_{6} x_{1}^{3}+a_{7} x_{1}^{2} x_{2}+a_{8} x_{1} x_{2}^{2}+a_{9} x_{2}^{3}=0 .
\end{aligned}
$$

We will write $K$ simultaneously for the cubic $K$ in $\mathbb{P}^{2}$, a cubic polynomial giving $K$ in terms of the coordinates $\left(x_{0}: x_{1}: x_{2}\right)$, and the corresponding point $K \in \mathbb{P}^{9}$. Similarly, $\lambda \in \breve{\mathbb{P}}^{2}$ will stand for both the line $\lambda$ in $\mathbb{P}^{2}$ and a corresponding linear function in terms of $\left(x_{0}: x_{1}: x_{2}\right)$.

We observed already (see $\S 1$ ) that the point-conditions $P_{0}$ in $\mathbb{P}^{9}$ are hyperplanes, while the line-conditions $L_{0}$ form hypersurfaces of degree 4. Explicitly, the line-condition corresponding to the line $x_{0}=0$ is the discriminant of the polynomial in $x_{1}, x_{2}$

$$
a_{6} x_{1}^{3}+a_{7} x_{1}^{2} x_{2}+a_{8} x_{1} x_{2}^{2}+a_{9} x_{2}^{3}
$$

hence has equation

$$
a_{7}^{2} a_{8}^{2}+18 a_{6} a_{7} a_{8} a_{9}-4 a_{6} a_{8}^{3}-4 a_{7}^{3} a_{9}-27 a_{6}^{2} a_{9}^{2}=0 .
$$

The following facts about line-conditions are independent of the corresponding line, therefore can be checked on $(*)$ :

Lemma 0.1. Let $L$ be the line-condition in $\mathbb{P}^{9}$ corresponding to $\lambda \in \check{\mathbb{P}}^{2}$. Then:

(1) If $K \in L$, then $L$ is smooth at $K$ if and only if $K$ intersects $\lambda$ with multiplicity exactly 2 at a point. In particular, the line-conditions are generically smooth along the locus $S_{0}$ of nonreduced cubics.

(2) If $K$ intersects $\lambda$ with multiplicity 3 at a point, then $L$ has multiplicity 2 at $K$. In particular, the line-conditions have multiplicity 2 along the locus $B_{0}$ of triple lines.

(3) The tangent hyperplane to $L$ at a smooth point $K$ consists of the cubics containing the point of tangency of $K$ to $\lambda$. The tangent cone in $V_{0}=\mathbb{P}^{9}$ to $L$ at a cubic $K$ intersecting $\lambda$ in a triple point $p$ is supported on the hyperplane in $V_{0}$ consisting of the cubics containing $p$.

The intersection of all line-conditions consists of the locus of non-reduced cubics, which we denote $S_{0} . S_{0}$ is the image of the 1-1 morphism

$$
\begin{gathered}
\check{\mathbb{P}}^{2} \times \check{\mathbb{P}}^{2} \stackrel{\phi_{0}}{\longrightarrow} \mathbb{P}^{9} \\
(\lambda, \mu) \mapsto \lambda \mu^{2}
\end{gathered}
$$

which maps the pair of lines with equations $\{\lambda=0\},\{\mu=0\}$ to the cubic of equation $\left\{\lambda \mu^{2}=0\right\}$. If $\Delta$ is the diagonal in $\check{\mathbb{P}}^{2} \times \check{\mathbb{P}}^{2}, \phi_{0}(\Delta)$ is the locus $B_{0}$ of triple lines. 
Lemma 0.2. The restriction of $\phi_{0}: \check{\mathbb{P}}^{2} \times \check{\mathbb{P}}^{2}-\Delta \rightarrow S_{0}-B_{0}$ is an isomorphism. In particular, $S_{0}-B_{0}$ is nonsingular.

Proof. The locus where $d \phi_{0}$ is an isomorphism is clearly invariant under projective transformations of $\mathbb{P}^{2}$, and the group of projective transformations acts transitively on $\check{\mathrm{P}}^{2} \times \check{\mathrm{P}}^{2}-\Delta$.

In fact, $S_{0}$ is singular along $B_{0}$ (see Remarks 1.4); we choose $B_{0}$ as the center of the first blow-up.

Lemma 0.3. $\quad B_{0}$ is the third Veronese imbedding of $\check{\mathbb{P}}^{2}$ in $\mathbb{P}^{9}$, thus a nonsingular 2-dimensional subvariety of $V_{0}$. The tangent space to $B_{0}$ in $V_{0}$ at a point $\lambda^{3} \in B_{0}$ consists of the cubics vanishing twice along $\lambda$.

Proof. $\phi_{0}$ restricts on $\check{\mathbb{P}}^{2} \cong \Delta \rightarrow B_{0}$ to $\lambda \mapsto \lambda^{3}$, the $3^{\text {rd }}$ Veronese imbedding $v_{3}$. The last assertion is checked by differentiating $\lambda \mapsto \lambda^{3}$.

We can get now the information needed for Theorem III (1):

Theorem III (1). (i) The intersection ring of $B_{0} \cong \mathbb{P}^{2}$ is generated by the hyperplane class $h$;

(ii) $c\left(N_{B_{0}} V_{0}\right)=(1+3 h)^{10} /(1+h)^{3}$.

Proof. (i) is just setting the notation;

(ii) the class of the hyperplane in $V_{0} \cong \mathbb{P}^{9}$ pulls-back to $3 h$ on $B_{0}$, and $c\left(N_{B_{0}}\right)=j_{0}^{*} c\left(T \mathbb{P}^{9}\right) / c\left(T B_{0}\right)$.

We also find

Lemma 0.4. $j_{0}^{*} P_{0}=3 h, j_{0}^{*} L_{0}=12 h$; the full intersection classes of a pointconditions and line-conditions with respect to $B_{0}$ are

$$
B_{0} \circ P_{0}=3 h, \quad B_{0} \circ L_{0}=2+12 h .
$$

Proof. The pull-back of the hyperplane class from $V_{0}$ to $B_{0}$ is $3 h . B_{0}$ is not contained in any point-condition, and the line-conditions have multiplicity 2 along $B_{0}$ by 0.1 .

3.1 The first blow-up. Let $V_{1}=\mathrm{B} l_{B_{0}} V_{0}$, write $\pi_{1}: V_{1} \rightarrow V_{0}$ for the blow-up map, $E_{1}$ for the exceptional divisor, and denote by $S_{1}, P_{1}, L_{1}$ the proper transforms of $S_{0}, P_{0}, L_{0}$. Then $P_{1}=\pi_{1}^{*} P_{0}, L_{1}=\pi_{1}^{*} L_{0}-2 E_{1}$ as divisor classes.

We will see here that the line-conditions in $V_{1}$ intersect along the smooth 4-dimensional proper transform $S_{1}$ of $S_{0}$ and along a smooth 4-dimensional subvariety $B_{1}$ of the exceptional divisor $E_{1}$ (Proposition 1.2). We will choose $B_{1}$ as the center for the second blow-up.

We determine now the intersection of the line-conditions in $V_{1}$. Since $V_{1}-E_{1} \cong V_{0}-B_{0}, S_{1}$ must be a component of the intersection. To find components contained in $E_{1}$, identify $E_{1}$ with the projective bundle $\mathbb{P}\left(N_{B_{0}} V_{0}\right)$ 
over $B_{0}$; the key observation is

Lemma 1.1. There is an imbedding $N_{v_{2}\left(\dot{\mathbb{P}}_{2}\right)} \mathbb{P}^{5} \hookrightarrow N_{v_{3}\left(\dot{\mathbb{P}}^{2}\right)} \mathbb{P}^{9}$ of vector bundles over $\check{P}^{2}$.

Proof. We have the exact sequences on $B_{0} \cong \check{\mathbb{P}}^{2}=\mathbb{P}(\check{Q})$

$$
\begin{gathered}
0 \rightarrow \mathscr{O}_{\dot{\mathbf{p}}^{2}} \rightarrow \check{Q} \otimes \mathscr{O}_{\dot{\mathbf{p}}^{2}}(1) \rightarrow T \check{\mathrm{P}}^{2} \rightarrow 0, \\
0 \rightarrow \mathscr{O}_{\dot{\mathbf{p}}^{2}} \rightarrow \operatorname{Sym}^{2} \check{Q} \otimes \mathscr{O}_{\dot{\mathbf{p}}^{2}}(2) \rightarrow T \mathrm{P}^{5} \rightarrow 0, \\
0 \rightarrow \mathscr{O}_{\dot{\mathbf{p}}^{2}} \rightarrow \operatorname{Sym}^{3} \check{Q} \otimes \mathscr{O}_{\dot{\mathbf{p}}^{2}}(3) \rightarrow T \mathbb{P}^{9} \rightarrow 0:
\end{gathered}
$$

the first is the standard Euler sequence on $\mathbb{P} \check{Q}$; the second and third are the pullbacks of the Euler sequences on $\mathbb{P}\left(\operatorname{Sym}^{2} \check{Q}\right)$ and $\mathbb{P}\left(\operatorname{Sym}^{3} \check{Q}\right)$ via the Veronese imbeddings $v_{2}: \check{\mathbb{P}}^{2} \rightarrow \mathbb{P}^{5}$ and $v_{3}: \check{\mathbb{P}}^{2} \rightarrow \mathbb{P}^{9}$. From these we get

$$
N_{v_{2}\left(\dot{\mathbf{P}}^{2}\right)} \mathbb{P}^{5}=\frac{\operatorname{Sym}^{2} \check{Q} \otimes \mathscr{O}(2)}{\check{Q} \otimes \mathscr{O}(1)}, \quad N_{v_{3}\left(\dot{\boldsymbol{P}}^{2}\right)} \mathbb{P}^{9}=\frac{\operatorname{Sym}^{3} \check{Q} \otimes \mathscr{O}(3)}{\check{Q} \otimes \mathscr{O}(1)} .
$$

The claimed imbedding $N_{v_{2}\left(\dot{\mathbb{P}}^{2}\right)} \mathbb{P}^{5} \hookrightarrow N_{v_{3}\left(\dot{\mathbb{P}}^{2}\right)} \mathbb{P}^{9}$ is induced by the map

$$
\operatorname{Sym}^{2} \check{Q} \otimes \mathscr{O}(-1) \rightarrow \operatorname{Sym}^{2} \check{Q} \otimes \check{Q} \rightarrow \operatorname{Sym}^{3} \check{Q}:
$$

this gives an imbedding

$$
\operatorname{Sym}^{2} \check{Q} \otimes \mathscr{O}(2)=\operatorname{Sym}^{2} \check{Q} \otimes \mathscr{O}(-1) \otimes \mathscr{O}(3) \hookrightarrow \operatorname{Sym}^{3} \check{Q} \otimes \mathscr{O}(3),
$$

that induces an imbedding on the quotients.

We can then define a 4-dimensional smooth subvariety $B_{1}$ of $E_{1}$, i.e.

$$
B_{1}=\mathbb{P}\left(N_{v_{2}\left(\dot{\boldsymbol{P}}^{2}\right)} \mathbb{P}^{5}\right) \hookrightarrow \mathbb{P}\left(N_{v_{3}\left(\dot{\mathbb{P}}^{2}\right)} \mathbb{P}^{9}\right)=E_{1} .
$$

Notice that the fiber of $\operatorname{Sym}^{2} \check{Q} \otimes \mathscr{O}(-1) \hookrightarrow \operatorname{Sym}^{3} \check{Q}$ over $\lambda \in \mathbb{P}(\check{Q})$ consists of the cubic polynomials over $Q$ divisibie by $\lambda$. The fiber of $B_{1}=\mathbb{P}\left(N_{v_{2}\left(\dot{P}^{2}\right)} \mathbb{P}^{5}\right)$ is then $\mathbb{P}\left(T R_{\lambda} / T B_{0}\right)$, where $R_{\lambda} \cong \mathbb{P}^{5}$ is the subspace of $V_{0}$ consisting of the cubics vanishing along (i.e. containing) the line $\lambda$. Also recall (Lemma 0.3) that the tangent space to $B_{0}$ in $\mathbb{P}^{9}$ consists of the cubics vanishing twice along $\lambda$. The information carried by a point of $B_{1}$ consists then of a line $\lambda$ and of the web of conics with given proper intersection with $\lambda$ : i.e., of $\lambda$ and of two points on $\lambda$. Of course these are the 'complete conics' supported on a double line: $\mathbb{P}\left(N_{v_{2}\left(\mathscr{P}_{2}\right)} \mathbb{P}^{5}\right)$ is the exceptional divisor in the space of complete conics (cf. [CX, 2.2]). We will refer to points of $B_{1}$ as to 'lines with distinguished pairs of points'. 
Proposition 1.2. The set-intersection of all line-conditions in $V_{1}$ is contained in the union of the smooth 4-folds $S_{1}$ and $B_{1}$.

Proof. $B_{1}$ is a projective bundle over $\mathbb{P}^{2}$, therefore it is smooth; the smoothness of $S_{1}$ is proved in Lemma 1.3 below.

We have to show that the line-conditions intersect along $B_{1}$ over $B_{0}$, and this can be checked fiberwise. As observed above, the fiber of $B_{1}$ over a triple line $\mu^{3} \in B_{0}$ is $\mathbb{P}\left(T_{\mu^{3}} R_{\mu} / T_{\mu^{3}} B_{0}\right)$, where $R_{\mu} \cong \mathbb{P}^{5}$ is the subspace of $V_{0}$ consisting of the cubics containing $\mu$; on the other hand, by Lemma 0.1 the intersection of the tangent cones to the line-conditions (in $\mathrm{P}^{9}$ ) at $\mu^{3} \in B_{0}$ is precisely $R_{\mu}$, so the assertion follows.

$B_{1}$ will be the center for the next blow-up.

Theorem III (2). $B_{1}$ is a $\mathbb{P}^{2}$-bundle over $B_{0}$.

(i) The intersection ring of $B_{1}$ is generated by the pull-back $h$ of $h$ via $B_{1} \rightarrow B_{0}$ and the pull-back $\varepsilon$ of $E_{1}$ via $j_{1}: B_{1} \hookrightarrow V_{1}$, and

$\int_{B_{1}} h^{4}=0, \quad \int_{B_{1}} h^{3} \varepsilon=0, \quad \int_{B_{1}} h^{2} \varepsilon^{2}=1, \quad \int_{B_{1}} h \varepsilon^{3}=9, \quad \int_{B_{1}} \varepsilon^{4}=51$

(ii) $c\left(N_{B_{1}} V_{1}\right)=(1+\varepsilon)(1+3 h-\varepsilon)^{10} /(1+2 h-\varepsilon)^{6}$.

Proof. (i) The universal line bundle on $\mathbb{P}\left(N_{v_{2}\left(\check{\mathbf{P}}^{2}\right)} \mathbb{P}^{5}\right)=B_{1}$ is the restriction of the one on $\mathbb{P}\left(N_{v_{3}\left(\dot{\boldsymbol{P}}^{2}\right)} \mathbb{P}^{9}\right)=E_{1}$, i.e. $\varepsilon$ (Lemma 1.1). The first assertion follows then from [F, Example 8.3.4]. Moreover, via $B_{1} \rightarrow B_{0}, 1-\varepsilon+\varepsilon^{2}-\varepsilon^{3}+\varepsilon^{4}$ pushes forward to

$$
s\left(N_{\dot{v}_{2}\left(\dot{P}_{2}\right)} \mathbb{P}^{5}\right)=\frac{(1+h)^{3}}{(1+2 h)^{6}}=1-9 h+51 h^{2}
$$

by [F, Chapter 4], so that the relations follow from the projection formula.

(ii) The normal bundle to $B_{1}$ in $V_{1}$ is an extension of $N_{B_{1}} E_{1}$ and $N_{E_{1}} V_{1}$. By Lemma 1.1, there is a commutative diagram

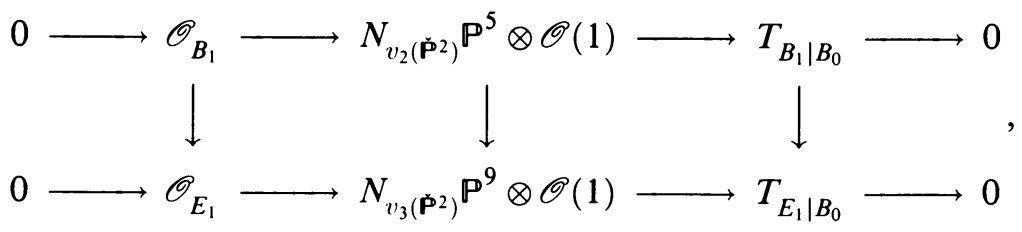

so that

$$
c\left(N_{B_{1}} E_{1}\right)=\frac{c\left(N_{v_{3}\left(\dot{\mathbf{P}}_{2}\right)} \mathbb{P}^{9} \otimes \mathscr{O}(1)\right)}{c\left(N_{v_{2}\left(\dot{\mathbf{P}}^{2}\right)} \mathbb{P}^{5} \otimes \mathscr{O}(1)\right.}=\frac{(1+3 h-\varepsilon)^{10}}{(1+2 h-\varepsilon)^{6}} .
$$

On the other hand, $c\left(N_{E_{1}} V_{1}\right)=1+\varepsilon$; thus (ii) follows from the Whitney product formula.

We proceed next to a closer analysis of the varieties involved at this stage. 
Consider the triple line $x_{0}^{3} \in B_{0} \hookrightarrow V_{0}=\mathbb{P}^{9}$. Setting $a_{0}=1$, affine coordinates for $V_{0}$ at $x_{0}^{3}$ are $\left(a_{1}, \ldots, a_{9}\right)$, and

$$
\begin{array}{rlr}
3 a_{3}-a_{1}^{2}=0, & 3 a_{4}-2 a_{1} a_{2}=0, \quad 3 a_{5}-a_{2}^{2}=0, \\
9 a_{6}-a_{1} a_{3}=0, & 3 a_{7}-a_{2} a_{3}=0, \quad 3 a_{8}-a_{1} a_{5}=0, \\
9 a_{9}-a_{2} a_{5}=0, &
\end{array}
$$

are equations for $B_{0}$ in a neighborhood of $x_{0}^{3}$. Thus we can choose coordinates $\left(b_{1}, \ldots, b_{9}\right)$ in an open in $V_{1}=\mathrm{B} l_{B_{0}} V_{0}$ so that

$$
\begin{aligned}
& b_{1}=a_{1} \text {, } \\
& b_{2}=a_{2} \text {, } \\
& b_{3}=3 a_{3}-a_{1}^{2}, \\
& b_{4} b_{3}=3 a_{4}-2 a_{1} a_{2} \text {, } \\
& b_{5} b_{3}=3 a_{5}-a_{2}^{2} \text {, } \\
& b_{6} b_{3}=9 a_{6}-a_{1} a_{3} \text {, } \\
& b_{7} b_{3}=3 a_{7}-a_{2} a_{3} \text {, } \\
& b_{8} b_{3}=3 a_{8}-a_{1} a_{5} \text {, } \\
& b_{9} b_{3}=9 a_{9}-a_{2} a_{5} \text {. }
\end{aligned}
$$

With this choice, $b_{3}=0$ is the equation for the exceptional divisor $E_{1}$, and $\left(b_{4}, \ldots, b_{9}\right)$ are coordinates for the fiber of $E_{1}$ over a point of $B_{0}$.

Recall $(\S 3.0)$ that $S_{0}$ is the image of a 1-1 morphism $\phi_{0}: \check{\mathbb{P}}^{2} \times \check{\mathbb{P}}^{2} \rightarrow \mathbb{P}^{9}$, which restricts to an isomorphism on $\check{\mathbb{P}}^{2} \times \check{\mathbb{P}}^{2}-\Delta$, where $\Delta$ is the diagonal in $\check{\mathbb{P}}^{2} \times \check{\mathbb{P}}^{2}$.

Lemma 1.3. The proper transform $S_{1}$ of $S_{0}$ is nonsingular, in fact isomorphic to $B l_{\Delta} \check{\mathscr{P}}^{2} \times \check{\mathbb{P}}^{2}$.

Proof. We will show that $\phi_{0}$ lifts to an isomorphism $\phi_{1}: \mathrm{B} l_{\Delta} \check{\mathbb{P}}^{2} \times \check{\mathbb{P}}^{2} \rightarrow S_{1}$ compatible with $\phi_{0}$; that is, such that the following diagram commutes:

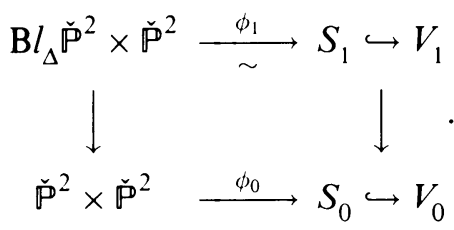

Let $e$ be the exceptional divisor of $\mathrm{B} l_{\Delta} \check{\mathbb{P}}^{2} \times \check{\mathbb{P}}^{2}$. A morphism $\phi_{1}$ exists by the universal property of blow-ups, and restricts to an isomorphism on $\mathrm{B} l_{\Delta} \check{\mathrm{P}}^{2} \times \check{\mathbb{P}}^{2}$ $-e$ by Lemma $0.2(2)$; so we only need to check that $\left(d \phi_{1}\right)_{p}$ is injective for $p \in e$. This matter is local and invariant under projective transformations of $\mathbb{P}^{2}$, so we can assume $p$ is in the fiber of $\left(x_{0}, x_{0}\right) \in \Delta$. Choose local coordinates $\left(\alpha_{1}, \alpha_{2} ; u_{1}, u_{2}\right)$ at $\left(x_{0}, x_{0}\right)$ so that $\left(\alpha_{1}, \alpha_{2} ; u_{1}, u_{2}\right)$ corresponds to

$$
\left(x_{0}+\left(\alpha_{1}+u_{1}\right) x_{1}+\left(\alpha_{2}+u_{2}\right) x_{2}, x_{0}+\alpha_{1} x_{1}+\alpha_{2} x_{2}\right) \in \check{\mathbb{P}}^{2} \times \check{\mathbb{P}}^{2} .
$$

Equations for $\Delta$ are then $u_{1}=0, u_{2}=0$. Therefore, we can choose coordinates $\left(\alpha_{1}, \alpha_{2} ; u, t\right)$ in an open set (that we can assume contains $p$ ) in $\mathrm{B} l_{\Delta} \check{\mathrm{P}}^{2} \times \check{\mathrm{P}}^{2}$ so that

$$
\alpha_{1}=a_{1}, \quad \alpha_{2}=a_{2}, \quad u=u_{1}, \quad u t=u_{2} ;
$$

the equation for $e$ is $u=0$. 
By the commutativity of $(*)$, in terms of the coordinates given for $V_{1}$ and these coordinates for $\mathrm{B} l_{\Delta} \check{\mathrm{P}}^{2} \times \check{\mathrm{P}}^{2}, \phi_{1}$ is given explicitly by

$$
(* *)\left(\alpha_{1}, \alpha_{2} ; u, t\right) \mapsto\left(3 \alpha_{1}+u, 3 \alpha_{2}+u t,-u^{2}, 2 t, t^{2}, 2 \alpha_{1}, 2 \alpha_{1} t, 2 \alpha_{2} t, 2 \alpha_{2} t^{2}\right),
$$

with nondegenerate jacobian, as needed.

Remarks 1.4.

(1) We will let $e$ denote the exceptional divisor in $\mathrm{B} l_{\Delta} \check{\mathrm{P}}^{2} \times \check{\mathbb{P}}^{2}$ (and its divisor class).

(2) $\phi_{1}^{*} E_{1}=2 e: S_{1}$ is tangent to $E_{1}$ along $e$. Consequently, $S_{0}$ has multiplicity 2 along $B_{0}$ : it is indeed singular along it.

(3) A point in $e$ can be visualized as a 'double line with distinguished point'. As a pair of lines $(\lambda, \mu) \in \check{\mathbb{P}}^{2} \times \check{\mathbb{P}}^{2}$ approaches an element $(\nu, \nu) \in \Delta$ along some curve, their intersection $\lambda \cap \mu$ approaches a specific point on $\nu$. Elements in $e$ record this information. If $\left(\alpha_{1}, \alpha_{2}, u, t\right)$ $\notin e$ (i.e. if $u \neq 0$ ), then the corresponding pair of lines intersects in the point $\left(\alpha_{1} t-\alpha_{2}:-t: 1\right)$; if $\left(\alpha_{1}, \alpha_{2}, 0, t\right) \in e$, then $\left(\alpha_{1} t-\alpha_{2}:-t: 1\right)$ are the coordinates of the 'distinguished point' on the line $x_{0}+\alpha_{1} x_{1}+$ $\alpha_{2} x_{2}=0$.

Lemma 1.5. (1) $B_{1}$ intersects $S_{1}$ along $e$.

(2) The line conditions in $V_{1}$ are generically smooth and tangent to $E_{1}$ along $B_{1}$.

Proof. (1) This is easily checked using the explicit expression $(* *)$ for $\phi_{1}$ in the proof of Lemma 1.3. By invariance under projective transformations, we can assume $\lambda=x_{0}$; equations for $R_{\lambda}$ are then $a_{6}=a_{7}=a_{8}=a_{9}=0$, and the check is immediate.

(2) By the invariance under projective transformations, it is enough to verify the claim for the line-condition corresponding to $x_{2}=0$, and we can restrict to the open set on which our local coordinates for $V_{1}$ hold. In terms of these coordinates, the proper transform to the line-condition has equation

$$
4 b_{3}+\left(3 b_{6}-2 b_{1}\right)^{2}=0 \text {, }
$$

and the assertion is easily checked.

Lemma 1.6. $j_{1}^{*} P_{1}=3 h, j_{1}^{*} L_{1}=12 h-2 \varepsilon$. The full intersection classes of pointand line-conditions with respect to $B_{1}$ are

$$
B_{1} \circ P_{1}=3 h, \quad B_{1} \circ L_{1}=1+12 h-2 \varepsilon .
$$

Proof. $P_{1}=\pi_{1}^{*} P_{0}, L_{1}=\pi_{1}^{*} L_{0}-2 E_{1} ; P_{1}$ does not contain $B_{1}$, and $e_{B_{1}} L_{1}=1$ follows from Lemma 1.5 (2).

Remarks 1.7.

(1) In terms of the descriptions of $e$ and $B_{1}, \phi_{1}$ acts on $e$ by mapping the line $\lambda$ with the distinguished point $p$ into the triple line $\lambda$ with the 
distinguished double point $p$. Therefore, $\phi_{1}$ maps the fiber of $e$ over $\lambda$ to a nonsingular conic in the fiber of $B_{1}$ over $\lambda^{3}$.

(2) Using the last remark and $(* *)$ in the proof of 1.3 , one gets equations for $B_{1}$ in terms of the local coordinates in $V_{1}$ :

$$
\begin{aligned}
& b_{3}=0, \quad 3 b_{6}-2 b_{1}=0, \quad 3 b_{7}-b_{1} b_{4}=0, \\
& 3 b_{8}-b_{2} b_{4}=0, \quad 3 b_{9}-2 b_{2} b_{5}=0 \text {. }
\end{aligned}
$$

(3) Lemma 1.5 (2) can be stated more precisely:

Consider a point $\tilde{\lambda} \in B_{1}$, i.e. a line $\lambda$ with distinguished points $p_{1}, p_{2}$.

Then the line-condition in $V_{1}$ corresponding to a line $\mu$ is nonsingular at $\tilde{\lambda}$ if $p_{1} \notin \mu$ and $p_{2} \notin \mu$.

The check is again immediate, for the equation of the proper transform of the line-condition corresponding to $x_{2}=0$.

3.2. The second blow-up. Let $V_{2}=\mathrm{B} l_{B_{1}} V_{1}$, write $\pi_{2}: V_{2} \rightarrow V_{1}$ for the blowup map, $E_{2}$ for the exceptional divisor, and denote by $\widetilde{E}_{1}, S_{2}, P_{2}, L_{2}$ the proper transforms of $E_{1}, S_{1}, P_{1}, L_{1}$. Then $P_{2}=\pi_{2}^{*} P_{1}$, and $L_{2}=\pi_{2}^{*} L_{1}-E_{2}$ (Lemma 1.5 (2)).

In $V_{2}$, we will see that the line-conditions intersect in the proper transform $S_{2}$ of $S_{1}$ and in a smooth 7-dimensional subvariety $B_{2}$ of the exceptional divisor $E_{2}$ (Proposition 2.1). $B_{2}$ will be the new center of blow-up.

Set $B_{2}=\widetilde{E}_{1} \cap E_{2} . B_{2}$ is the exceptional divisor of the blow-up of $E_{1}$ at $B_{1}$, therefore (see $\S 3.1$ )

$$
B_{2}=\mathbb{P}\left(N_{B_{1}} E_{1}\right)=\mathbb{P}\left(\frac{\operatorname{Sym}^{3} \check{Q} \otimes \mathscr{O}(3)}{\operatorname{Sym}^{2} \check{Q} \otimes \mathscr{O}(2)} \otimes \mathscr{O}_{B_{1}}(1)\right)
$$

is a $\mathbb{P}^{3}$-bundle over $B_{1}$. In particular, $B_{2}$ is smooth.

Proposition 2.1. The set-intersection of all line-conditions in $V_{2}$ is contained in the union of $S_{2}$ and the 7-dimensional smooth variety $B_{2}=\widetilde{E}_{1} \cap E_{2}$.

Proof. $V_{2}-E_{2} \cong V_{1}-B_{1}$, thus $S_{2}$ is a component of the intersection. By Lemma 1.5 (2), the line-conditions in $V_{1}$ are generically tangent to $E_{1}$, so their proper transforms all intersect $E_{2}$ along $\widetilde{E}_{1} \cap E_{2}=B_{2}$.

The center for the next blow-up will be $B_{2}$.

Theorem III (3). $B_{2}$ is a $\mathbb{P}^{3}$-bundle on $B_{1}$.

(i) The intersection ring of $B_{2}$ is generated by the pull-backs $h, \varepsilon$ of $h, \varepsilon$ via the projection $B_{2} \rightarrow B_{1}$, and the pull-back $\varphi=j_{2}^{*} E_{2}$ of $E_{2}$ via 


$$
\begin{aligned}
& j_{2}: B_{2} \rightarrow V_{2} \text {. Also, } \\
& \int_{B_{2}} \varphi^{7}=-210, \quad \int_{B_{2}} \varphi^{6} h=-90, \quad \int_{B_{2}} \varphi^{6} \varepsilon=-240, \\
& \int_{B_{2}} \varphi^{5} h^{2}=-10, \quad \int_{B_{2}} \varphi^{5} h \varepsilon=0, \quad \int_{B_{2}} \varphi^{5} \varepsilon^{2}=105 \text {, } \\
& \int_{B_{2}} \varphi^{4} h^{2} \varepsilon=4, \quad \int_{B_{2}} \varphi^{4} h \varepsilon^{2}=18, \quad \int_{B_{2}} \varphi^{4} \varepsilon^{3}=42 ， \\
& \int_{B_{2}} \varphi^{3} h^{2} \varepsilon^{2}=-1, \quad \int_{B_{2}} \varphi^{3} h \varepsilon^{3}=-9, \quad \int_{B_{2}} \varphi^{3} \varepsilon^{4}=-51,
\end{aligned}
$$

hold (all other codimension-7 terms have degree 0 ).

(ii) $c\left(N_{B_{2}} V_{2}\right)=(1+\varphi)(1+\varepsilon-\varphi)$.

Proof. (i) $B_{2}=\mathbb{P}\left(N_{B_{1}} E_{1}\right)$, with universal line bundle induced from $E_{2}=$ $\mathbb{P}\left(N_{B_{1}} V_{1}\right)$, so the first assertion follows. Moreover, $1-\varphi+\varphi^{2}-\varphi^{3}+\varphi^{4}-$ $\varphi^{5}+\varphi^{6}-\varphi^{7}$ pushes forward to

$$
s\left(N_{B_{1}} E_{1}\right)=\frac{(1+2 h-\varepsilon)^{6}}{(1+3 h-\varepsilon)^{10}},
$$

and the relations follow directly by Lemma 1.7 and the projection formula.

(ii) $B_{2}=E_{2} \cap \widetilde{E}_{1}$, so that $c\left(N_{B_{2}} V_{2}\right)=c\left(N_{E_{2}} V_{2}\right) c\left(N_{\widetilde{E}_{1}} V_{2}\right)$.

We now obtain a more detailed description of the situation, for future reference. As for $S_{2}$ :

Lemma 2.1. $S_{2}$ is isomorphic to $S_{1}$, hence to $B l_{\Delta} \check{\mathrm{P}}^{2} \times \check{\mathrm{P}}^{2}$.

Proof. By Lemma 1.5 (1), $S_{2}$ is the blow-up of $S_{1}$ along a divisor, thus it is isomorphic to $S_{1}$.

A description of $B_{2}$ is gotten as follows:

Let $\tilde{\lambda}$ be a point in $B_{1}$ mapping to $\lambda^{3} \in B_{0}$ via $B_{1} \rightarrow B_{0}$ (i.e. ' $\lambda$ with two distinguished points'). The fiber of $B_{2}$ above $\tilde{\lambda}$ can be identified with the space $\mathbb{P}\left(\left(\operatorname{Sym}^{3} \check{Q}\right)_{\grave{\lambda}} /\left(\operatorname{Sym}^{2} \check{Q}\right)_{\grave{\lambda}}\right)$, where $\left(\operatorname{Sym}^{2} \check{Q}\right)_{\grave{\lambda}} \hookrightarrow\left(\operatorname{Sym}^{3} \check{Q}\right)_{\grave{\lambda}}$ is the multiplication by $\lambda \cdot \mathbb{P}\left(\left(\operatorname{Sym}^{3} \check{Q}\right)_{\bar{\lambda}} /\left(\operatorname{Sym}^{2} \check{Q}\right)_{\grave{\lambda}}\right)$ is the 3-dimensional space of cubics on $\lambda$ : a point in the fiber of $B_{2}$ above $\tilde{\lambda}$ corresponds then to a triple of points on $\lambda$, and we will refer to points of $B_{2}$ as to lines with a pair of distinguished points and a triple of distinguished points.

Lemma 2.2. (1) $B_{2}$ intersects $S_{2} \cong B l_{\Delta} \check{\mathscr{P}}^{2} \times \check{\mathbb{P}}^{2}$ along $e$.

(2) The line-conditions in $V_{2}$ are generically smooth along $B_{2}$.

Proof. (1) Recall that $S_{1}$ is tangent to $E_{1}$ along $e$ (Remark 1.4 (2)). Thus $S_{2} \cap E_{2} \subset \widetilde{E}_{1} \cap E_{2}=B_{2}$.

(2) By Lemma $1.5(2)$, the line-conditions in $V_{1}$ are generically smooth along $B_{1}$. 
This gives us the additional information about conditions we will need in the computation in $\S 4$ :

Lemma 2.3. $j_{2}^{*} P_{2}=3 h, j_{2}^{*} L_{2}=12 h-2 \varepsilon-\varphi$. The full intersection classes for point- and line-conditions with respect to $B_{2}$ are

$$
B_{2} \circ P_{2}=3 h \quad, \quad B_{2} \circ L_{2}=1+12 h-2 \varepsilon-\varphi .
$$

Proof. $P_{2}=\pi_{2}^{*} P_{1} ; L_{2}=\pi_{2}^{*} L_{1}-E_{2} . P_{2}$ does not contain $B_{2}$, and $e_{B_{2}} L_{2}=1$ follows from Lemma $2.2(2)$.

In the local coordinates given for $V_{1}$ in $\S 3.1$, equations for $B_{1}$ are

$$
\begin{aligned}
b_{3}=0, \quad 3 b_{6}-2 b_{1} & =0, \quad 3 b_{7}-b_{1} b_{4}=0, \\
3 b_{8}-b_{2} b_{4} & =0, \quad 3 b_{9}-2 b_{2} b_{5}=0,
\end{aligned}
$$

(Remark $1.7(2))$ thus we can choose coordinates $\left(c_{1}, \ldots, c_{9}\right)$ in an open set in $V_{2}$ so that

$$
\begin{aligned}
& c_{1}=b_{1}, \\
& c_{2}=b_{2}, \quad c_{3} c_{6}=b_{3} \text {, } \\
& c_{4}=b_{4} \text {, } \\
& c_{5}=b_{5} \text {, } \\
& c_{6}=3 b_{6}-2 b_{1} \text {, } \\
& c_{7} c_{6}=3 b_{7}-b_{1} b_{4} \text {, } \\
& c_{8} c_{6}=3 b_{8}-b_{2} b_{4}, \\
& c_{9} c_{6}=3 b_{9}-2 b_{2} b_{5} \text {. }
\end{aligned}
$$

In the coordinates $\left(c_{1}, \ldots, c_{9}\right)$, equations for $E_{2}$ and $\widetilde{E}_{1}$ are $c_{6}=0$ and $c_{3}=0$ respectively.

Recall that $S_{1}$ is the isomorphic image of a map $\phi_{1}: \mathrm{B} l_{\Delta} \check{\mathbb{P}}^{2} \times \check{\mathbb{P}}^{2} \hookrightarrow V_{1}$ (see Lemma 1.3) given in local coordinates by

$$
\left(\alpha_{1}, \alpha_{2} ; u, t\right) \mapsto\left(3 \alpha_{1}+u, 3 \alpha_{2}+u t,-u^{2}, 2 t, t^{2}, 2 \alpha_{1}, 2 \alpha_{1} t, 2 \alpha_{2} t, 2 \alpha_{2} t^{2}\right) .
$$

Now $\phi_{1}$ lifts to a map $\phi_{2}: \mathrm{B} l_{\Delta} \check{\mathrm{P}}^{2} \times \check{\mathrm{P}}^{2} \hookrightarrow V_{2}$; a local coordinate expression for $\phi_{2}$ is

$$
\left(\alpha_{1}, \alpha_{2} ; u, t\right) \mapsto\left(3 \alpha_{1}+u, 3 \alpha_{2}+u t, \frac{u}{2}, 2 t, t^{2},-2 u, t, t^{2}, t^{3}\right) .
$$

Remark 2.4.

(1) From this it follows $\phi_{2}^{*} E_{2}=e$.

(2) Using $(*)$, one checks that in terms of the descriptions of $e$ as set of lines with distinguished point, and of $B_{2}$ as set of lines with distinguished pair and triple of points, $\phi_{2}$ acts $e \rightarrow B_{2}$ by mapping the line $\lambda$ with distinguished point $p$ to the line $\lambda$ with distinguished double point $p$ and triple point $p$.

Lemma 2.5. Let $\bar{\lambda}$ be a point on $B_{2}$, i.e. a line $\lambda$ with distinguished pair of points $p_{1}, p_{2}$ and triple of points $q_{1}, q_{2}, q_{3}$, and consider the line-condition $L_{\mu}$ in $V_{2}$ corresponding to a line $\mu \neq \lambda$. Then:

(1) $L_{\mu}$ is tangent to $E_{2}$ at $\bar{\lambda}$ if $\exists i, p_{i} \in \mu$;

(2) $L_{\mu}$ is tangent to $\widetilde{E}_{1}$ at $\bar{\lambda}$ if $\exists i, q_{i} \in \mu$. 
Proof. We can assume $\lambda=x_{0}, \mu=x_{1}$, by invariance under projective transformations. In local coordinates, the equation for $L_{\mu}$ is then

$$
4 c_{3} c_{5}^{3}+c_{6} c_{9}^{2}=0
$$

and coordinates for $\bar{\lambda}$ have $c_{5}=0$ if the pair touches $\mu, c_{9}=0$ if the triple touches $\mu$. The verifications are immediate.

3.3. The third blow-up. Let $V_{3}=\mathrm{B} l_{B_{2}} V_{2}$, write $\pi_{3}: V_{3} \rightarrow V_{2}$ for the blow-up map, $E_{3}$ for the exceptional divisor, and denote by $S_{3}, P_{3}, L_{3}$ the proper transforms of $S_{2}, P_{2}, L_{2}$. Then $P_{3}=\pi_{3}^{*} P_{2}$, and $L_{3}=\pi_{3}^{*} L_{2}-E_{3}$ (Lemma $2.2(2))$.

In $V_{3}$ the line-conditions will intersect in the proper transform $S_{3}$ of $S_{2}$, a 4-dimensional smooth variety isomorphic to the blow-up of $\check{\mathrm{P}}^{2} \times \check{\mathrm{P}}^{2}$ along the diagonal (Lemma 3.1, Proposition 3.2). We will choose $S_{3}$ as the center $B_{3}$ for the fourth blow-up.

We first of all remark:

Lemma 3.1. $S_{3}$ is isomorphic to $S_{2}$, hence to $B l_{\Delta} \check{\mathrm{P}}^{2} \times \check{\mathrm{P}}^{2}$.

Proof. By Lemma $2.2(1), S_{3}$ is the blow-up of $S_{2}$ along a divisor.

Then

Proposition 3.2. The intersection of all line-conditions in $V_{3}$ is supported on the 4-dimensional smooth irreducible variety $S_{3}$.

Proof. We have to verify that the line-conditions intersect in $E_{3}$ only along $S_{3} \cap E_{3}$. Since $B_{2}$ has codimension 2 in $V_{2}, E_{3}$ is a $\mathbb{P}^{1}$-bundle over $B_{2}$. A general line-condition is smooth at $\bar{\lambda} \in B_{2}$ (Lemma 2.2 (2)), thus the lineconditions in $V_{3}$ can intersect in at most one point over each $\bar{\lambda} \in B_{2}$. We have then to check that the line-conditions in $V_{3}$ can intersect in $E_{3}$ only above $B_{2} \cap S_{2}$, i.e. only above $\bar{\lambda} \in B_{2}$ with coincident pair and triple of points (see Remark 2.4 (2))

Notice that since $B_{2}=\widetilde{E}_{1} \cap E_{2}$, the proper transforms of $\widetilde{E}_{1}, E_{2}$ in $V_{3}$ cut the fiber of $E_{3}$ over any $\bar{\lambda} \in B_{2}$ in distinct points, say $r_{1}, r_{2}$. Fix now $\bar{\lambda} \in B_{2}$, i.e. a line $\lambda$ with distinguished pair $p_{1}, p_{2}$ and triple $q_{1}, q_{2}, q_{3}$, and let $\mu \neq \lambda$ be a line. As a consequence of Lemma 2.5:

if $\mu$ touches the pair, then the line-condition in $V_{3}$ corresponding to $\mu$ contains $r_{2}$;

if $\mu$ touches the triple, then the line-condition in $V_{3}$ corresponding to $\mu$ contains $r_{1}$.

We conclude that the line-conditions can intersect over $\bar{\lambda}$ only if $p_{1}=p_{2}=$ $q_{1}=q_{2}=q_{3}$, i.e. if $\bar{\lambda} \in B_{2} \cap S_{2}$.

Therefore in $V_{3}$ the line-conditions intersect along the smooth and irreducible 4-dimensional variety $S_{3} \cong \mathrm{B} l_{\Delta} \check{P}^{2} \times \check{\mathbb{P}}^{2}$. We choose $S_{3}$ as the center for the next blow-up: in other words, we let $B_{3}$ be $S_{3}$. 
Note that $B_{3} \cong \mathrm{B} l \breve{P}^{2} \times \check{\mathbb{P}}^{2}$ has two natural projections onto $\check{\mathbb{P}}^{2}$ : let $l, m$ be the pull-backs via these projections of the hyperplane class in $\check{\mathbb{P}}^{2}$, and denote by $e$ the exceptional divisor.

Theorem III (4). $B_{3} \cong B l_{\Delta} \check{\mathrm{P}}^{2} \times \check{\mathbb{P}}^{2}$.

(i) The intersection ring of $B_{3}$ is generated by $l, m, e$, and the relations $e m=e l, l^{3}=m^{3}=0$,

$$
\begin{array}{ll}
\int_{B_{3}} l^{2} m^{2}=1, & \int_{B_{3}} e^{2} l^{2}=-1, \\
\int_{B_{3}} e^{3} l=-3, & \int_{B_{3}} e^{4}=-6 ;
\end{array}
$$

(ii) $c\left(N_{B_{3}} V_{3}\right)=1+7 l+17 m-16 e+126 m^{2}+99 l m+21 l^{2}-315 e l+105 e^{2}+$ $582 l m^{2}+237 l^{2} m-2517 e l^{2}+1611 e^{2} l-358 e^{3}+1026 l^{2} m^{2}+9174 e^{2} l^{2}-$ $3912 e^{3} l+652 e^{4}$.

Proof. (i) Call $k$ the hyperplane class in $\check{\mathbb{P}}^{2} \cong \Delta \stackrel{\delta}{\hookrightarrow} \check{\mathbb{P}}^{2} \times \check{\mathbb{P}}^{2}$. Since $k=\delta^{*} l=$ $\delta^{*} m$, then $l, m, e$ generate the intersection ring of $B_{3}$ (cf. [F, Example 8.3.9]). $e m=e l$ is clear, while the other relations are checked observing that $e-e^{2}+$ $e^{3}-e^{4}$ pushes forward to

$$
s\left(\Delta, \check{\mathbb{P}}^{2} \times \check{\mathbb{P}}^{2}\right)=\frac{1}{(1+k)^{3}} .
$$

(ii) $j_{3}^{*} c\left(T V_{3}\right), c\left(T B_{3}\right)$ can be obtained by applying the blow-up Chern classes formula (cf. [F, Theorem 15.4]).

Then $c\left(N_{B_{3}} V_{3}\right)$ is computed as $j_{3}^{*} c\left(T V_{3}\right) / c\left(T B_{3}\right)$.

In the local coordinates given for $V_{2}$ in $\S 3.2$, equations for $B_{2}$ are

$$
c_{3}=0, \quad c_{6}=0
$$

(recall that $B_{2}=\widetilde{E}_{1} \cap E_{2}$ ), thus we can choose coordinates $\left(d_{1}, \ldots d_{9}\right)$ in an open set in $V_{3}$ such that

$$
\begin{aligned}
& d_{1}=c_{1}, \quad d_{2}=c_{2}, \quad d_{3}=c_{3}, \\
& d_{4}=c_{4}, \quad d_{5}=c_{5}, \quad d_{6} d_{3}=c_{6}, \\
& d_{7}=c_{7}, \quad d_{8}=c_{8}, \quad d_{9}=c_{9} .
\end{aligned}
$$

The equation of the exceptional divisor is $d_{3}=0$.

The map $\phi_{2}: \mathrm{B} l_{\Delta} \check{\mathbb{P}}^{2} \times \check{\mathbb{P}}^{2} \rightarrow V_{2}$ lifts to a map $\phi_{3}: \mathrm{B} l_{\Delta} \check{\mathbb{P}}^{2} \times \check{\mathbb{P}}^{2} \rightarrow V_{3}$, given in coordinates by

$$
\left(\alpha_{1}, \alpha_{2} ; u, t\right) \mapsto\left(3 \alpha_{1}+u, 3 \alpha_{2}+u t, u / 2,2 t, t^{2},-4, t, t^{2}, t^{3}\right) .
$$

Lemma 3.3. $j_{3}^{*} P_{3}=l+2 m, j_{3}^{*} L_{3}=4 l+8 m-6 e$; the full intersection classes for points- and line-conditions with respect to $B_{3}$ are

$$
B_{3} \circ P_{3}=l+2 m \quad, \quad B_{3} \circ L_{3}=1+4 l+8 m-6 e .
$$


Proof. $j_{3}^{*} P_{3}=l+2 m$ because of the commutativity of the diagram

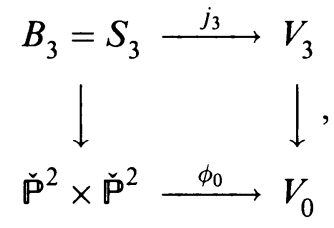

by the definition of $\phi_{0}$ in $\S 3.0$, and since $P_{3}$ is the pull-back of a hyperplane from $V_{0} \cdot j_{3}^{*} L_{3}=4 l+8 m-6 e$ because $L_{3}=\pi_{3}^{*} L_{2}-E_{3}, L_{2}=\pi_{2}^{*} L_{1}-E_{2}$, $L_{1}=\pi_{1}^{*} L_{0}-2 E_{1}, L_{0}$ is a hypersurface of degree 4 in $V_{0}$, and $\phi_{1}^{*} E_{1}=2 e(\mathrm{Re}-$ mark $1.4(2)), \phi_{2}^{*} E_{2}=e$ (Remark $\left.2.4(1)\right)$, and $\phi_{3}^{*} E_{3}=e$. No point-conditions in $V_{3}$ contain $B_{3}$, therefore $e_{B_{3}} P_{3}=0 ; e_{B_{3}} L_{3}=1$ follows from Lemma 0.1 (1), since $V_{0}$ and $V_{3}$ are isomorphic away from $B_{0}$ and from the exceptional divisors.

Remarks 3.4.

(1) The equations of the line-conditions in $V_{3}$ corresponding to lines through the point $(1: 0: 0)$ are written in terms of $d_{4}, \ldots, d_{9}$ only, as seen by direct computation.

(2) On the other hand, the last six coordinates $d_{4}, \ldots, d_{9}$ of the image of a point via $\phi_{3}$ are constant along divisors $\{t=$ const. $\}$ in $\mathrm{B} l \breve{P}^{2} \times \check{\mathbb{P}}^{2}$.

Therefore, the behavior of line-conditions corresponding to lines containing (1:0:0) is constant along the sets $\{t=$ const. $\}$ in $\mathrm{B} l_{\Delta} \check{\mathrm{P}}^{2} \times \breve{\mathrm{P}}^{2}$. For example, to check the transversality of line-conditions corresponding to lines through (1:0:0) at all points of the image of $\mathrm{B} l_{\Delta} \check{\mathscr{P}}^{2} \times \check{\mathbb{P}}^{2}$, it is enough to check it for points not contained in $e$. This argument will be applied in $\S \S 3.4,3.5$; it can also be used to give a second proof of Proposition 3.2.

3.4. The fourth blow-up. Let $V_{4}=\mathrm{B} l_{B_{3}} V_{3}$, write $\pi_{4}: V_{4} \rightarrow V_{3}$ for the blowup map, $E_{4}$ for the exceptional divisor, and denote by $P_{4}, L_{4}$ the proper transforms of $P_{3}, L_{3}$. Then $P_{4}=\pi_{4}^{*} P_{3}$, and $L_{4}=\pi_{4}^{*} L_{3}-E_{4}$ (Lemma 0.1 (1)).

In $V_{4}$, the line-conditions will still intersect in a 4-dimensional smooth variety $B_{4}$, contained in $E_{4}$ (Proposition 4.1 ). $\quad B_{4}$ will be the last center of blow-up.

Proposition 4.1. The intersection of all line-conditions in $V_{4}$ is a smooth 4dimensional subvariety $B_{4}$ of $E_{4}=\mathbb{P}\left(N_{B_{3}} V_{3}\right)$. More precisely, $B_{4}=\mathbb{P}(\mathscr{L})$, $\mathscr{L}$ a subline bundle of $N_{B_{3}} V_{3}$.

Proof. First consider a point in $B_{3} \cong \mathrm{B} l_{\Delta} \check{\mathbb{P}}^{2} \times \check{\mathbb{P}}^{2}$ not on the exceptional divisor $e$. There exist isomorphic neighborhoods of such a point in $V_{3}$ and of a point $\lambda \mu^{2} \in S_{0}, \lambda \neq \mu$, in $V_{0}=\mathbb{P}^{9}$; by Lemma 0.1 (3) the tangent hyperplanes to the line-conditions in $V_{0}$ at $\lambda \mu^{2}$ intersect in the 5-dimensional subspace 
$R_{\mu}$ of $V_{0}$ consisting of the cubics containing $\mu$. In fact, if $\lambda, \mu$ do not contain $(1: 0: 0)$ it is enough to consider line-conditions corresponding to lines containing $(1: 0: 0)$.

The tangent space to $R_{\mu}$ at $\lambda \mu^{2}, T_{\lambda \mu^{2}} R_{\mu}$, contains the 4-dimensional $T_{\lambda \mu^{2}} S_{0}$; as $\lambda, \mu$ vary, $T_{\lambda \mu^{2}} R_{\mu} / T_{\lambda \mu^{2}} S_{0}$ determine a line-bundle $\mathscr{L}^{\circ}$ over $S_{0}-B_{0} \cong$ $B_{3}-e$, and the intersection of the line-conditions in $V_{4}$ above points in $B_{3}$ outside e is supported on $\mathbb{P}\left(\mathscr{L}^{\circ}\right) \hookrightarrow \mathbb{P}\left(N_{B_{3}} V_{3}\right)=E_{4}$. By Remarks 3.4, $\mathscr{L}^{\circ}$ extends to a line bundle $\mathscr{L}$ over the whole $B_{3}$, and the line-conditions intersect along $\mathbf{P}(\mathscr{L})$ as claimed.

We choose $B_{4}$ for the next (and last) center of blow-up: let $j_{4}: B_{4} \hookrightarrow V_{4}$ be the inclusion. The next lemma gives the information needed to compute $c\left(N_{B_{4}} V_{4}\right)$.

Lemma 4.2. $c_{1}(\mathscr{L})=3 l+3 m-4 e$.

Proof. $\mathbb{P}(\mathscr{L})$ is isomorphic to $B_{3}=\mathrm{B} l_{\Delta} \check{\mathrm{P}}^{2} \times \check{\mathbb{P}}^{2}$ via the projection map. To compute $c_{1}(\mathscr{L})$, notice that the restriction of $\mathscr{O}(-1)$ from $\mathbb{P}\left(N_{B_{3}} V_{3}\right)=E_{4}$ to $\mathbb{P}(\mathscr{L})$ is the pull-back of $\mathscr{L}$, so that, via the isomorphism $\mathbb{P}(\mathscr{L}) \rightarrow B_{3}$, $c_{1}(\mathscr{L})=j_{4}^{*} E_{4}$.

Consider then the divisor $F_{0}$ in $V_{0}$ with equation

$$
\left|\begin{array}{ccc}
3 a_{0} & a_{1} & a_{2} \\
2 a_{1} & 2 a_{3} & a_{4} \\
2 a_{2} & a_{4} & 2 a_{5}
\end{array}\right|=12 a_{0} a_{3} a_{5}-3 a_{0} a_{4}^{2}-4 a_{1}^{2} a_{5}+4 a_{1} a_{2} a_{4}-4 a_{2}^{2} a_{3}=0 .
$$

The rows of the determinant are coefficients of second partial derivatives of the equation of a cubic, therefore it is clear that this divisor contains $S_{0}$ (the cubics in $S_{0}$ have a triple point). If $F_{1}, F_{2}, F_{3}$ denote the proper transforms of $F_{0}$ in $V_{1}, V_{2}, V_{3}$, one checks that $F_{1}=\pi_{1}^{*} F_{0}-2 E_{1}, F_{2}=\pi_{2}^{*} F_{1}, F_{3}=\pi_{3}^{*} F_{2}$. Since $F_{0}$ has degree 3 , it follows that $j_{3}^{*} F_{3}=3 l+6 m-4 e$. Now $F_{0}$ has multiplicity 1 along $S_{0}$ : thus $F_{3}$ has multiplicity 1 along $B_{3}$, and if $F_{4}$ is the proper transform of $F_{3}$ in $V_{4}$ we get

$$
c_{1}(\mathscr{L})=3 l+6 m-4 e-j_{4}^{*} F_{4} .
$$

By the description of $\mathscr{L}$ given in the proof of Proposition 4.1, $F_{4}$ meets $B_{4}=\mathbb{P}(\mathscr{L})$ at a point mapping to $\lambda \mu^{2} \in V_{0}-B_{0}$ if the tangent hyperplane to $F_{0}$ at $\lambda \mu^{2}$ contains the space $R_{\mu}$ of cubics containing $\mu$. Using this fact, one computes $j_{4}^{*} F_{4}=3 m$, getting

$$
c_{1}(\mathscr{L})=3 l+6 m-4 e-3 m=3 l+3 m-4 e
$$

as needed.

Note that $B_{4}$ is isomorphic to $B_{3}=\mathrm{B} l_{\Delta} \check{\mathrm{P}}^{2} \times \check{\mathrm{P}}^{2}$ via the projection $\mathbb{P}(\mathscr{L}) \rightarrow$ $B_{3}$; thus its intersection ring is generated by the pull-backs of $l, m, e$, which we will still denote $l, m, e$, with the relations stated in Theorem III (4): 
Theorem III (5). $B_{4} \cong B_{3}$.

(i) The intersection ring of $B_{4}$ is generated by $l, m, e$, and em $=e l$, $l^{3}=m^{3}=0$,

$$
\begin{array}{ll}
\int_{B_{3}} l^{2} m^{2}=1, & \int_{B_{3}} e^{2} l^{2}=-1, \\
\int_{B_{3}} e^{3} l=-3, & \int_{B_{3}} e^{4}=-6 ;
\end{array}
$$

(ii) $c\left(N_{B_{4}} V_{4}\right)=1-5 l+5 m+18 m^{2}-27 l m+3 l^{2}+21 e l-7 e^{2}-30 l m^{2}+$ $75 l^{2} m-225 e l^{2}+135 e^{2} l-30 e^{3}+75 l^{2} m^{2}$.

Proof. (i) is noticed above.

(ii) The Euler sequence

$$
0 \rightarrow \mathscr{O}_{E_{4}} \rightarrow N_{B_{3}} V_{3} \otimes \mathscr{O}(1) \rightarrow T_{E_{4} \mid B_{3}} \rightarrow 0
$$

restricts to

$$
0 \rightarrow \mathscr{O}_{\mathbf{P}(\mathscr{L})} \rightarrow N_{B_{3}} V_{3} \otimes \check{\mathscr{L}} \rightarrow T_{E_{4} \mid B_{3}} \rightarrow 0
$$

on $\mathbb{P}(\mathscr{L})$ (for ease of reading, we have suppressed the pull-back signs). Since $B_{4}=\mathbb{P}(\mathscr{L}) \cong B_{3}$ via the projection, it follows

$$
c\left(N_{B_{4}} E_{4}\right)=c\left(T_{E_{4} \mid B_{3}}\right)=c\left(N_{B_{3}} V_{3} \otimes \check{\mathscr{L}}\right),
$$

so that

$$
c\left(N_{B_{4}} V_{4}\right)=j_{4}^{*} c\left(N_{E_{4}} V_{4}\right) c\left(N_{B_{4}} E_{4}\right)=c(\mathscr{L}) c\left(N_{B_{3}} V_{3} \otimes \check{\mathscr{L}}\right),
$$

and (ii) follows.

Lemma 4.3. $j_{4}^{*} P_{4}=l+2 m, j_{4}^{*} L_{4}=l+5 m-2 e$. The full intersection classes for the point- and line-conditions with respect to $B_{4}$ are

$$
B_{4} \circ P_{4}=l+2 m, \quad B_{4} \circ L_{4}=1+l+5 m-2 e .
$$

Proof. $P_{4}=\pi_{4}^{*} P_{3}$ implies $j_{4}^{*} P_{4}=l+2 m$. The restriction of $\mathscr{O}(-1)$ from $E_{4}=\mathbb{P}\left(N_{B_{3}} V_{3}\right)$ to $\mathbb{P}(\mathscr{L})$ is the pull-back of $\mathscr{L}$, so $j_{4}^{*} L_{4}=j_{4}^{*}\left(\pi_{4}^{*} L_{3}-E_{4}\right)=$ $4 l+8 m-6 e-c_{1}(\mathscr{L})=l+5 m-2 e$ (Lemma 4.2). The point-conditions in $V_{4}$ do not contain $B_{4}$; the line-conditions in $V_{4}$ are generically smooth along $B_{4}$ since the line-conditions in $V_{3}$ are generically smooth along $B_{3}$.

3.5. The fifth blow-up. Let $V_{5}=\mathrm{B} l_{B_{4}} V_{4}$, write $\pi_{5}: V_{5} \rightarrow V_{4}$ for the blowup map, $E_{5}$ for the exceptional divisor, denote by $\widetilde{E}_{4}, P_{5}, L_{5}$ the proper transforms of $E_{4}, P_{4}, L_{4}$.

Finally, we will see that the line-conditions 'separate' in $V_{5}$ (Proposition 5.3), concluding the proof of Theorem III.

Consider $\widetilde{E}_{4} \cap E_{5}=\mathbb{P}\left(N_{B_{4}} E_{4}\right)$. 
Denote by $\mathscr{O}_{1}(-1)$ (resp. $\left.\mathscr{O}_{2}(-1)\right)$ the pull-back of the universal line-bundle from the first (resp. second) factor of $\check{\mathbb{P}}^{2} \times \check{\mathbb{P}}^{2}$ to $\check{\mathbb{P}}^{2} \times \check{\mathbb{P}}^{2}$. Recalling that $B_{3}-$ $e \cong S_{0}-B_{0} \hookrightarrow \check{\mathbb{P}}^{2} \times \check{\mathbb{P}}^{2}$ (and omitting pull-backs for sake of notations)

$$
N_{B_{3}-e} V_{3} \cong N_{S_{0}-B_{0}} V_{0}=T \mathbb{P}^{9} / T \check{\mathbb{P}}^{2} \times \check{\mathbb{P}}^{2}
$$

if $\mathbb{P}^{2}=\mathbb{P} Q$, so that $\mathbb{P}^{9}=\mathbb{P}\left(\operatorname{Sym}^{3} \check{Q}\right)$, then $T \mathbb{P}^{9}$ is given by the Euler sequence

$$
0 \rightarrow \mathscr{O}_{\mathbf{P}^{9}} \rightarrow \operatorname{Sym}^{3} \check{Q} \otimes \mathscr{O}_{\mathbf{P}^{9}}(1) \rightarrow T \mathbb{P}^{9} \rightarrow 0,
$$

thus

$$
\begin{aligned}
N_{B_{3}-e} V_{3} & \cong\left(\operatorname{Sym}^{3} \check{Q} \otimes \mathscr{O}_{p_{9}}(1) / \mathscr{O}_{p^{9}}\right) / T \check{\mathbb{P}}^{2} \times \check{\mathbb{P}}^{2} \\
& \cong\left(\operatorname{Sym}^{3} \check{Q} \otimes \mathscr{O}_{1}(1) \otimes \mathscr{O}_{2}(2) / \mathscr{O}_{B_{3}-e}\right) / T \check{\mathbb{P}}^{2} \times \check{\mathbb{P}}^{2} .
\end{aligned}
$$

$N_{B_{4}} E_{4} \cong T_{E_{4} \mid B_{4}}$ is given by

$$
0 \rightarrow \mathscr{O}_{E_{4}} \rightarrow N_{B_{3}} V_{3} \otimes \mathscr{O}_{E_{4}}(1) \rightarrow T_{E_{4} \mid B_{4}} \rightarrow 0
$$

restricting on $B_{4}=\mathbb{P}(\mathscr{L})$ to

$$
0 \rightarrow \mathscr{O}_{B_{4}} \rightarrow N_{B_{3}} V_{3} \otimes \check{\mathscr{L}} \rightarrow N_{B_{4}} E_{4} \rightarrow 0 .
$$

On the other hand, over $B_{3}-e$ the line bundle $\mathscr{L}$ restricts to

$$
\begin{aligned}
\grave{\mathscr{L}} & \cong\left(\operatorname{Sym}^{2} \check{Q} \otimes \mathscr{O}_{\mathrm{P} s}(1) / \mathscr{O}_{\mathrm{P}^{5}}\right) / T \check{\mathrm{P}}^{2} \times \check{\mathbb{P}}^{2} \\
& \cong\left(\operatorname{Sym}^{2} \check{Q} \otimes \mathscr{O}_{1}(1) \otimes \mathscr{O}_{2}(1) / \mathscr{O}_{B_{3}-e}\right) / T \check{\mathbb{P}}^{2} \times \check{\mathbb{P}}^{2}
\end{aligned}
$$

(this follows from the description of $\stackrel{\circ}{\mathscr{L}}$ given in the proof of Proposition 4.1). By $(*)$ and $(* *)$,

$$
N_{B_{4}} E_{4} \cong\left(N_{B_{3}} V_{3} \otimes \check{\mathscr{L}}\right) / \mathscr{O}_{B_{4}} \cong\left(N_{B_{3}} V_{3} / \mathscr{L}\right) \otimes \check{\mathscr{L}}
$$

restricts over $B_{4}-e$ to

$$
\frac{\operatorname{Sym}^{3} \check{Q}}{\operatorname{Sym}^{2} \check{Q} \otimes \mathscr{O}_{2}(-1)} \otimes \mathscr{O}_{1}(1) \otimes \mathscr{O}_{2}(2) \otimes \check{\mathscr{L}} .
$$

Therefore, over a point in $B_{4}-e$, mapping to $\lambda \mu^{2} \in S_{0}-\Delta$, the fiber of $N_{B_{4}} E_{4}$ can be identified with the space $\operatorname{Sym}^{3} \check{Q} / \operatorname{Sym}^{2} \check{Q}$, where the inclusion $\operatorname{Sym}^{2} \check{Q} \hookrightarrow \operatorname{Sym}^{3} \check{Q}$ is given by the multiplication by $\mu$. This space is canonically isomorphic to the space of homogeneous degree-3 polynomials on the line with equation $\mu=0$; consequently, a point of $\widetilde{E}_{4} \cap E_{5}=\mathbb{P}\left(N_{B_{4}} E_{4}\right)$ over $\lambda \mu^{2}$, $\lambda \neq \mu$, can be pictured as a cubic consisting of a line and a double line, with three distinguished points on the double line.

For $\lambda \neq \mu$ lines in $\mathbb{P}^{2}$, consider a point $\overline{\lambda \mu^{2}} \in \widetilde{E}_{4} \cap E_{5}$, i.e. $\lambda \mu^{2}$ 'with a distinguished triple of points specified on $\mu^{\prime}$, and a line $\nu \subset \mathbb{P}^{2}$. 
Lemma 5.1. Suppose $\nu$ does not contain $\lambda \cap \mu$. Then the line-condition in $V_{5}$ corresponding to $\nu$ contains $\overline{\lambda \mu^{2}}$ if and only if $\nu$ contains a point of the triple on $\mu$.

Proof. Let $L_{i}$ be the line-condition in $V_{i}$ corresponding to $\nu$. Since $\nu \nsupseteq \lambda \cap \mu$, then $L_{0}$ is nonsingular at $\lambda \mu^{2}$ by Lemma 0.1 (1). Let $H_{p} \subset \operatorname{Sym}^{3} \check{Q}$ denote the space of cubic polynomials on $Q$ vanishing at $p$. As $\lambda \mu^{2}$ varies in $S_{0}$, the $H_{\nu \cap \mu}$ define a subbundle $\mathscr{H}$ of $\operatorname{Sym}^{3} \check{Q}$ over a neighborhood of $\lambda \mu^{2}$; notice that $\mathscr{H} \supset \operatorname{Sym}^{2} \check{Q} \otimes \mathscr{O}_{2}(-1)$. By Lemma 0.1 (3), the tangent space to $L_{0}$ at $\lambda \mu^{2}$ is contained in $T_{\lambda \mu^{2}} \mathbb{P}^{9}$ as the image of $\mathscr{H} \otimes \mathscr{O}_{\mathbf{P}}(1)$; tracing the argument preceding this lemma identifies then the fiber of $L_{5} \cap \widetilde{E}_{4} \cap E_{5}$ over $\lambda \mu^{2}$ with $\mathbb{P}\left(H_{\nu \cap \mu} / \operatorname{Sym}^{2} \check{Q}\right)$, the space of triples on $\mu$ touching $\nu$.

Remark 5.2. Notice that this lemma implies that the intersection of all lineconditions in $V_{5}$ must be disjoint from $\widetilde{E}_{4} \cap E_{5}$. We will prove that it is empty by showing that it must also be contained in $\widetilde{E}_{4} \cap E_{5}$.

Proposition 5.3. The intersection of all line-conditions in $V_{5}$ is empty.

Proof. The line-conditions can intersect only in $E_{5}$. By Remarks 3.4, it is enough to check that the intersection is empty above $B_{4}-e$; and since the matter is invariant under projective transfomations, it is enough to check that the intersection of all line-conditions in $V_{5}$ is empty over a single point $\lambda \mu^{2} \in$ $B_{4}$, with $\lambda \neq \mu$.

The fiber $\left(E_{5}\right)_{\lambda \mu^{2}}$ of $E_{5}=\mathbb{P}\left(N_{B_{4}} V_{4}\right)$ over $\lambda \mu^{2}$ is a 4-dimensional projective space $\mathbb{P}^{4}$. For $\nu \nsupseteq \lambda \cap \mu$, the association

$\nu$ line in $\mathbb{P}^{2} \mapsto\left(E_{5}\right)_{\lambda \mu^{2}} \cap$ line-condition in $V_{5}$ corresponding to $\nu$

determines a rational map $\check{\mathrm{P}}^{2} \longrightarrow \rightarrow \check{\mathbb{P}}^{4}$. Notice that by the nonsingularity of $\check{\mathrm{P}}^{2}$, this extends in codimension 1 , so it must be defined for at least all $\nu \neq \lambda, \mu$. Let then $\nu \supset \lambda \cap \mu, \nu \neq \lambda, \mu$, denote by $\lambda \mu^{2}$ also the point on $B_{4}$ over $\lambda \mu^{2} \in \mathbb{P}^{9}$, and write $L_{\nu^{\prime}}$ for the line-condition in $V_{4}$ corresponding to a line $\nu^{\prime} \nsupseteq \lambda \cap \mu$. A coordinate computation shows that as $\nu^{\prime}$ approaches $\nu$, its tangent space at $\lambda \mu^{2} \quad T_{\lambda \mu^{2}} L_{\nu^{\prime}}$ approaches $T_{\lambda \mu^{2}} E_{4}$ (as subspaces of $T_{\lambda \mu^{2}} V_{4}$ ). It follows that the image of $\nu$ under $\check{\mathbb{P}}^{2} \rightarrow \breve{\mathbb{P}}^{4}$ is the intersection of $\widetilde{E}_{4}$ with the fiber of $E_{5}$ : this implies that the intersection of all line-conditions in $V_{5}$ is included in $\widetilde{E}_{4} \cap E_{5}$. On the other hand, by Remark 5.2 the intersection must be disjoint from $\widetilde{E}_{4} \cap E_{5}$ : therefore, it must be empty.

A different proof of an equivalent statement can be found in [St, II, p. 146]. Proposition 5.3 concludes the proof of Theorem III: $\widetilde{V}=V_{5}$ is a smooth variety of complete cubics. By Corollary I, the number of smooth cubics containing 
$n_{p}$ points and tangent to $n_{l}$ lines in general position $\left(n_{p}+n_{l}=9\right)$ is then $\int_{V_{5}} P_{5}^{n_{p}} L_{5}^{n_{l}}$. In the next section we will apply Theorem II to compute these intersection numbers.

\section{COMPUTATION OF THE CHARACTERISTIC NUMBERS}

We work over an algebraically closed field of characteristic $\neq 2,3$. The notations for this section are those used in the statement of Theorem III: $V_{0}=$ $\mathbb{P}^{9}, V_{i}$ is the $i$ th blow-up, $B_{i}$ the center for the $i+1$ th blow-up, the intersection rings of the $B_{i}$ 's are generated by various subsets of $\{h, \varepsilon, \phi, l, m, e\}$, with the relations listed in Theorem III. Furthermore (as in the rest of $\S 3$ ) $P_{i}, L_{i}$ denote respectively the point- and line-conditions in $V_{i}$; we found in $\S 3$ that the full intersection classes of $P_{i}, L_{i}$ with respect to $B_{i}, i=0, \ldots, 4$, are respectively

$$
\begin{array}{ll}
B_{0} \circ P_{0}=3 h, & B_{0} \circ L_{0}=2+12 h, \\
B_{1} \circ P_{1}=3 h, & B_{1} \circ L_{1}=1+12 h-2 \varepsilon, \\
B_{2} \circ P_{2}=3 h, & B_{2} \circ L_{2}=1+12 h-2 \varepsilon-\varphi, \\
B_{3} \circ P_{3}=l+2 m, & B_{3} \circ L_{3}=1+4 l+8 m-6 e, \\
B_{4} \circ P_{4}=l+2 m, & B_{4} \circ L_{4}=1+l+5 m-2 e .
\end{array}
$$

Also, Theorem III lists the total Chern classes $c\left(N_{B_{i}} V_{i}\right)$ and the relations in dimension 0 in the Chow groups of the $B_{i}$ 's. Therefore, the following statement translates the computation of the characteristic numbers of a family $F$ into the computation of a degree and of five full intersection classes $B_{i} \circ F_{i}$ :

Theorem IV (Notations of Theorem III). Let $F$ be an r-dimensional subvariety in $\mathbb{P}^{9}$ parametrizing a family of reduced cubics, and let $F_{i}$ be the proper transform in $V_{i}$ of the closure $F_{0}$ of $F$. Also, let $f$ be the degree of the closure of $F$. Then the number $N_{F}\left(n_{p} P, n_{l} L\right)$ of elements (counted with multiplicities) of $F$ containing $n_{p}$ given points and tangent to $n_{l}$ given lines in general position, with $n_{p}+n_{l}=r$, is

$$
N_{F}\left(n_{p} P, n_{l} L\right)=4^{n_{l}} \cdot f-\sum_{i=0}^{4} \int_{B_{i}} \frac{\left(B_{i} \circ P_{i}\right)^{n_{p}}\left(B_{i} \circ L_{i}\right)^{n_{l}}\left(B_{i} \circ F_{i}\right)}{c\left(N_{B_{i}} V_{i}\right)} .
$$

Furthermore, the elements containing the given points and properly tangent to the given lines are counted with multiplicity 1.

Proof. This follows from

$$
\begin{gathered}
\int_{V_{0}} P_{0}^{n_{p}} L_{0}^{n_{l}} F_{0}=4^{n_{l}} \cdot f \\
\int_{V_{i+1}} P_{i+1}^{n_{p}} L_{i+1}^{n_{l}} F_{i+1}=\int_{V_{i}} P_{i}^{n_{p}} L_{i}^{n_{l}} F_{i}-\int_{B_{l}} \frac{\left(B_{i} \circ P_{i}\right)^{n_{p}}\left(B_{i} \circ L_{i}\right)^{n_{l}}\left(B_{i} \circ F_{i}\right)}{c\left(N_{B_{i}} V_{i}\right)}, \\
N_{F}\left(n_{p} P, n_{l} L\right)=\int_{V_{5}} P_{5}^{n_{p}} L_{5}^{n_{l}} F_{5} .
\end{gathered}
$$


(1) follows from Bézout's Theorem, (2) from Theorem II, and (3) from Theorems I and III.

For the family $F$ of smooth cubics, we have $F_{i}=V_{i}$, so that $B_{i} \circ F_{i}=\left[B_{i}\right]$. Also, all tangencies are proper, thus the numbers given by Theorem IV are in fact the 'characteristic numbers'. Writing $N\left(n_{p} P, n_{l} L\right)=N_{F}\left(n_{p} P, n_{l} L\right)$ in this case, we get

Corollary IV. The characteristic numbers for the family of smooth plane cubics are given by

$$
N\left(n_{p} P, n_{l} L\right)=\left\{\begin{aligned}
1, & n_{p}=9, n_{l}=0, \\
4, & n_{p}=8, n_{l}=1, \\
16, & n_{p}=7, n_{l}=2, \\
64, & n_{p}=6, n_{l}=3, \\
256, & n_{p}=5, n_{l}=4, \\
976, & n_{p}=4, n_{l}=5, \\
3424, & n_{p}=3, n_{l}=6, \\
9766, & n_{p}=2, n_{l}=7, \\
21004, & n_{p}=1, n_{l}=8, \\
33616, & n_{p}=0, n_{l}=9 .
\end{aligned}\right.
$$

Proof. Theorem IV gives

$$
N\left(n_{p} P, n_{l} L\right)=4^{n_{l}}-\sum_{i=0}^{4} \int_{B_{i}} \frac{\left(B_{i} \circ P_{i}\right)^{n_{p}}\left(B_{i} \circ L_{i}\right)^{n_{l}}}{c\left(N_{B_{l}} V_{i}\right)}
$$

listing only the nonzero contributions, and understanding $n_{p}=9-n_{l}$ :

$$
\begin{array}{r}
\int_{B_{0}} \frac{(3 h)^{n_{p}}(2+12 h)^{n_{l}}(1+h)^{3}}{(1+3 h)^{10}}=\left\{\begin{aligned}
1152, & n_{l}=7 \\
16128, & n_{l}=8, \\
125952, & n_{l}=9,
\end{aligned}\right. \\
\int_{B_{1}} \frac{(3 h)^{n_{p}}(1+12 h-2 \varepsilon)^{n_{l}}(1+2 h-\varepsilon)^{6}}{(1+\varepsilon)(1+3 h-\varepsilon)^{10}}=\left\{\begin{aligned}
441, & n_{l}=7 \\
5229, & n_{l}=8, \\
32214, & n_{l}=9,
\end{aligned}\right. \\
\int_{B_{2}} \frac{(3 h)^{n_{p}}(1+12 h-2 \varepsilon-\varphi)^{n_{l}}}{(1+\varphi)(1+\varepsilon-\varphi)}=\left\{\begin{aligned}
2295, & n_{l}=7, \\
21411, & n_{l}=8, \\
97146, & n_{l}=9,
\end{aligned}\right.
\end{array}
$$




$$
\begin{gathered}
\int_{B_{3}} \frac{(l+2 m)^{n_{p}}(1+4 l+8 m-6 e)^{n_{l}}}{(1+7 l+17 m-16 e+\cdots)}=\left\{\begin{aligned}
24, & n_{l}=5, \\
390, & n_{l}=6, \\
1572, & n_{l}=7, \\
18, & n_{l}=8, \\
-22635, & n_{l}=9,
\end{aligned}\right. \\
\int_{B_{4}} \frac{(l+2 m)^{n_{p}}(1+l+5 m-2 e)^{n_{l}}}{(1-5 l+5 m+\cdots)}=\left\{\begin{aligned}
24, & n_{l}=5, \\
282, & n_{l}=6, \\
1158, & n_{l}=7, \\
1746, & n_{l}=8, \\
-4149, & n_{l}=9,
\end{aligned}\right.
\end{gathered}
$$

Each of these computations is performed by extracting the 0th dimensional terms in the series and using the relations in the rings of the $B_{i}$ 's listed in Theorem III. For example:

$$
\begin{aligned}
\int_{B_{4}} & \frac{(1+l+5 m-2 e)^{9}}{(1-5 l+5 m+\ldots)} \\
& =\int_{B_{4}} 48654 l^{2} m^{2}+126129 e^{2} l^{2}-29508 e^{3} l+2533 e^{4} \\
& =48654 \cdot 1+126129 \cdot(-1)-29508 \cdot(-3)+2533 \cdot(-6) \\
& =-4149 .
\end{aligned}
$$

The computations were carried out using Macsyma.

These results and $(*)$ above give $N\left(n_{p} P, n_{l} L\right)=4^{n_{l}}$ for $n_{l}=0, \ldots, 4$ and $n_{p}=9-n_{l}$, and

$$
N\left(n_{p} P, n_{l} L\right)=\left\{\begin{array}{l}
1024-0-0-0-24-24=976 \\
4096-0-0-0-390-282=3424 \\
16384-1152-441-2295-1572-1158=9766 \\
65536-16128-5229-21311-18-1746=21004 \\
262144-125952-32214-97146+22635+4149=33616
\end{array}\right.
$$

for $n_{l}=5, \ldots, 9$ as stated.

Corollary IV agrees with Maillard and Zeuthen's result. More generally, the relevant information needed to apply Theorem IV to a family $F$ is the behavior of the proper transforms $F_{i}$ of the closure $F_{0}$ of $F$, with respect to the $B_{i}$ 's. For example, if $F_{0}$ is a divisor of $\mathbb{P}^{9}$, all one needs is the degree of $F_{0}$ and the five multiplicities of the $F_{i}$ along the $B_{i}$ (for $F_{i}$ divisors, this information gives $\left.\left(B_{i} \circ F_{i}\right)\right)$. For example, for $F_{0}$ the divisor of singular cubics the multiplicities are $8,5,3,6,6$, as we will compute in a forthcoming note. These multiplicities and the degree of $F_{0}(12)$ are enough to compute the $N_{F}\left(n_{p} P, n_{l} L\right)$ for nodal cubics. From them, the characteristic numbers for nodal cubics will be obtained 
by further applications of Theorem IV to the families of nodal cubics with node on given line and node at a given point.

\section{A CODIMENSION-2 CONDITION}

Maillard and Zeuthen's results for smooth cubics go further than Corollary IV above. After computing the characteristic numbers involving point- and lineconditions, they list the numbers also involving the codimension- 2 conditions expressing tangency to a line at a given point.

Such conditions are linear: in a sense they are the intersection of two 'infinitely near' point-conditions. The numbers reflect this fact by agreeing with appropriate characteristic numbers from Corollary IV for low $n_{l}$; but as $n_{l}$ grows larger and nonreduced curves enter into the picture, their position with respect to the flag becomes relevant and one expects discrepancies to occur. It is natural to inquire whether the information we need to apply Theorem IV to the computation of the numbers involving codimension- 1 conditions is enough to obtain these other results; this is indeed the case, as we will show in this section.

The geometry of the situation is captured in five full intersection classes (Proposition 5.1); once they are computed, a statement analogous to Theorem IV gives the numbers involving these codimension-2 conditions for a family $F$ if the classes $B_{i} \circ F_{i}$ are known. As in $\S 4$, the application to the family of smooth plane cubics (over an algebraically closed field of characteristic $\neq 2,3$ ) is then immediate.

We will keep the style of the notations introduced in $\S 1$ : call point-lineconditions $M$ the linear subspaces $\mathbb{P}^{N-2} \hookrightarrow \mathbb{P}^{N}$ formed by the plane curves tangent to a given line at a given point; for any variety $\widetilde{V}$ mapping to $\mathbb{P}^{N}$, isomorphically over $\mathbb{P}^{N}-S$, call point-line-conditions in $\widetilde{V}$ the proper transforms $\widetilde{M}$ of the conditions $M$ of $\mathbb{P}^{N} . \widetilde{M}$ is regularly imbedded outside the inverse image of $S$ in $\widetilde{V}$; therefore, if the intersection of $\widetilde{M}$ with a subvariety $\widetilde{F}$ of $\widetilde{V}$ is proper and has no components lying over $S$, then the product $\widetilde{M} \cdot \widetilde{F}$ is defined.

Theorem $\mathrm{I}^{\prime}$. Let $\tilde{V}$ be a variety of complete curves of degree $d, F$ an $r$ dimensional subvariety in $\mathbb{P}^{N}$ parametrizing a family of reduced curves, and let $\widetilde{F}$ be the proper transform in $\widetilde{V}$ of the closure of $F$. Then the number of elements (counted with multiplicities) of $F$ containing $n_{p}$ given points, tangent to $n_{l}$ given lines, and tangent to $n_{m}$ given lines at specified points (all choices being general), with $n_{p}+n_{l}+2 n_{m}=r$, is $\widetilde{P}^{n_{p}} \cdot \widetilde{L}^{n_{l}} \cdot \widetilde{M}^{n_{m}} \cdot \widetilde{F}$. Furthermore, the elements satisfying the conditions and properly tangent to the lines are counted with multiplicity one.

Proof. We just sketch the arguments here, since they closely resemble those in $\S 1$. We also assume the notations and the basic set-up from $\S 1$. The main observation is the analogous for point-line-conditions of Lemma 1 in $\S 1$, namely: 
Claim. For $F \subset \mathbb{P}^{N}$, there exists a point-line-condition $M$ such that $\widetilde{M \cap F}=$ $\widetilde{M} \cap \widetilde{F}$.

Indeed, one has to check that $\widetilde{M} \cap \widetilde{F}$ does not have components over $S$. But a point-line-condition $M$ is contained in the intersection of the corresponding point-condition $P$ and line-condition $L$, so that $\widetilde{M} \cap \widetilde{F} \subset \widetilde{L} \cap \widetilde{P} \cap \widetilde{F}$. We can choose the point so that $\widetilde{P} \cap \widetilde{F}$ has no components over $S$ (Lemma 2 in $\S 1$, and for a general line through that point we can get $\widetilde{L} \cap \widetilde{P} \cap \widetilde{M}$ with no components over $S$ (the set of line-conditions corresponding to lines through a point is nondegenerate in $\check{\mathbb{P}}^{M}$ ).

The claim implies the first part of the theorem, by the same argument in the proof of Theorem I (1) in $\S 1$.

The proof of the statement about multiplicities is likewise similar to the proof of Theorem I (2) in $\S 1$.

Before stating Theorem IV', we compute the full intersection classes $B_{i} \circ M_{i}$, $i=0, \ldots, 4$, for point-line-conditions. Here the notations are those used in Theorem III, $M_{0}$ denotes a point-line-condition in $V_{0}=\mathbb{P}^{9}$, and $M_{i}$ is the proper-transform of $M_{i-1}$ in $V_{i}$ (i.e., a 'point-line-condition' in $V_{i}$ ).

Proposition 5.1 (Full intersection classes for point-line-conditions).

(1) $B_{0} \circ M_{0}=2 h+9 h^{2}$,

(2) $B_{1} \circ M_{1}=h+9 h^{2}-2 \varepsilon h$,

(3) $B_{2} \circ M_{2}=h+9 h^{2}-2 \varepsilon h-\phi h$,

(4) $B_{3} \circ M_{3}=m+l^{2}+4 l m+4 m^{2}-6 e l$,

(5) $B_{4} \circ M_{4}=m+l^{2}+l m+m^{2}-2 e l$.

Proof. The main tools are the geometry of the blow-ups ( $\S 3$ ), and (iii) from $\S 2$.

(1) $M_{0}$ is nonsingular, has codimension 2 and intersects $B_{0}$ along the pencil $\mathbb{P}^{1} \subset \mathbb{P}^{2}=B_{0}$ of triple lines through the given point; therefore $B_{0} \circ M_{0}=$ $\left[B_{0} \cap M_{0}\right]+B_{0} \cdot M_{0}$. An algebraic check gives $\left[B_{0} \cap M_{0}\right]=2 h$; and since the hyperplane in $\mathbb{P}^{9}=V_{0}$ pulls-back to $3 h$ on $B_{0}$, (1) follows.

(2) Notice that $M_{0}$ is contained in the line-condition $L_{0}$ corresponding to the given line, and in the point-condition $P_{0}$ corresponding to the point. The fiber of $M_{1}$ over a point of $B_{0} \cap M_{0}$ is 5-dimensional and contained into (therefore coinciding with) the irreducible 5-dimensional fiber of $L_{1}$ over the same point. It follows that $B_{1} \cap M_{1}$ is (set-theoretically) the fiber in $B_{1}$ of $B_{0} \cap M_{0}$. Also, $\operatorname{dim}\left(\operatorname{Sing} M_{1}\right) \leq \operatorname{dim} B_{0} \cap M_{0}=1$, thus $M_{1}$ is generically nonsingular along $B_{1} \cap M_{1}$; it follows $B_{1} \circ M_{1}=\left[B_{1} \cap M_{1}\right]+B_{1} \cdot M_{1}$. Since $M_{1} \subset L_{1} \cap P_{1}, L_{1}$ is generically nonsingular along $B_{1}$ (Lemma 1.5 (2) in $\S 3$ ), and $P_{1}$ cuts transversally $B_{1}$, then $\left[B_{1} \cap M_{1}\right]=h$. Finally, one applies Fulton's blow-up formula (Theorem 6.7 in [F]) to get $B_{1} \cdot M_{1}=9 h^{2}-2 \varepsilon h$, as stated.

(3), (4) and (5) are obtained using the same arguments.

Now we can state the extension of Theorem IV: 
Theorem IV' (Notations of Theorem III). Let $F$ an $r$-dimensional subvariety in $\mathbf{P}^{9}$ parametrizing a family of reduced cubics, and let $F_{i}$ be the proper transform in $V_{i}$ of the closure $F_{0}$ of $F$. Also, let $f$ be the degree of the closure of $F$. Then the number $N_{F}\left(n_{p} P, n_{l} L, n_{m} M\right)$ of elements (counted with multiplicities) of $F$ containing $n_{p}$ given points, tangent to $n_{l}$ given lines, and tangent to $n_{m}$ given lines at specified points (all choices being general), with $n_{p}+n_{l}+2 n_{m}=r$, is

$$
\begin{aligned}
N_{F}\left(n_{p} P\right. & \left., n_{l} L, n_{m} M\right) \\
& =4^{n_{l}} \cdot f-\sum_{i=0}^{4} \int_{B_{i}} \frac{\left(B_{i} \circ P_{i}\right)^{n_{p}}\left(B_{i} \circ L_{i}\right)^{n_{l}}\left(B_{i} \circ M_{i}\right)^{n_{m}}\left(B_{i} \circ F_{i}\right)}{c\left(N_{B_{i}} V_{i}\right)} .
\end{aligned}
$$

Furthermore, the elements containing the given points and properly tangent to the given lines are counted with multiplicity one.

Proof. Similarly to Theorem IV, this is a consequence of

$$
\begin{gathered}
\int_{V_{0}} P_{0}^{n_{p}} L_{0}^{n_{l}} M_{0}^{n_{m}} F_{0}=4^{n_{l}} \cdot f, \\
\int_{V_{i+1}} P_{i+1}^{n_{p}} L_{i+1}^{n_{l}} M_{i+1}^{n_{m}} F_{i+1}=\int_{V_{i}} P_{i}^{n_{p}} L_{i}^{n_{l}} M_{i}^{n_{m}} F_{i} \\
-\int_{B_{i}} \frac{\left(B_{i} \circ P_{i}\right)^{n_{p}}\left(B_{i} \circ L_{i}\right)^{n_{l}}\left(B_{i} \circ M_{i}\right)^{n_{m}}\left(B_{i} \circ F_{i}\right)}{c\left(N_{B_{i}} V_{i}\right)}, \\
N_{F}\left(n_{p} P, n_{l} L\right)=\int_{V_{5}} P_{5}^{n_{p}} L_{5}^{n_{l}} M_{5}^{n_{m}} F_{5},
\end{gathered}
$$

where now (3) follows from Theorem I' and III.

This applies immediately to the family of smooth cubics; denoting the numbers in this case by $N\left(n_{p} P, n_{l} L, n_{m} M\right)$ :

Corollary IV'.

$$
N\left(n_{p} P, n_{l} L, 1 M\right)=\left\{\begin{aligned}
1, & n_{p}=7, n_{l}=0, \\
4, & n_{p}=6, n_{l}=1, \\
16, & n_{p}=5, n_{l}=2, \\
64, & n_{p}=4, n_{l}=3, \\
244, & n_{p}=3, n_{l}=4, \\
856, & n_{p}=2, n_{l}=5, \\
2344, & n_{p}=1, n_{l}=6, \\
4726, & n_{p}=0, n_{l}=7 ;
\end{aligned}\right.
$$




$$
\begin{gathered}
N\left(n_{p} P, n_{l} L, 2 M\right)=\left\{\begin{aligned}
1, & n_{p}=5, n_{l}=0, \\
4, & n_{p}=4, n_{l}=1, \\
16, & n_{p}=3, n_{l}=2, \\
62, & n_{p}=2, n_{l}=3, \\
220, & n_{p}=1, n_{l}=4, \\
576, & n_{p}=0, n_{l}=5 ;
\end{aligned}\right. \\
N\left(n_{p} P, n_{l} L, 3 M\right)=\left\{\begin{array}{cc}
1, & n_{p}=3, n_{l}=0, \\
4, & n_{p}=2, n_{l}=1, \\
16, & n_{p}=1, n_{l}=2, \\
58, & n_{p}=0, n_{l}=3 ;
\end{array}\right. \\
N\left(n_{p} P, n_{l} L, 4 M\right)=\left\{\begin{array}{cc}
1, & n_{p}=1, n_{l}=0, \\
4, & n_{p}=0, n_{l}=1 .
\end{array}\right.
\end{gathered}
$$

Proof. This follows from Theorem IV' applied to the family of smooth cubics; in this case, $B_{i} \circ F_{i}=\left[B_{i}\right]$. We just list here the relevant contributions:

$$
\begin{aligned}
& N(\left.\left(7-n_{l}\right) P, n_{l} L, 1 M\right) \\
&= \begin{cases}256-0-0-0-6-6=244, & n_{l}=4, \\
1024-0-0-0-99-69=856, & n_{l}=5, \\
4096-384-147-765-240-216=2344, & n_{l}=6, \\
16384-4992-1596-6372+1287+15=4726, & n_{l}=7 ;\end{cases} \\
& N\left(\left(5-n_{l}\right) P, n_{l} L, 2 M\right) \begin{array}{ll}
64-0-0-0-1-1=62, & n_{l}=3, \\
256-0-0-0-21-15=220, & n_{l}=4, \\
1024-128-49-255+13-29=576, & n_{l}=5 ;
\end{array} \\
& N(0 P, 3 L, 3 M)=64-0-0-0-3-3=58 .
\end{aligned}
$$

Corollary IV' also agrees with Maillard and Zeuthen's results.

\section{REFERENCES}

[A] P. Aluff, The characteristic numbers for smooth plane cubics, Algebraic Geometry (Sundance, 1986), Lecture Notes in Math., vol. 1311, Springer, 1986.

[CX] E. Casas-Alvero and S. Xambó-Descamps, The enumerative theory of conics after Halphen, Lecture Notes in Math., vol. 1196, Springer, 1986.

[F] W. Fulton, Intersection theory, Springer-Verlag, 1984.

[KS] S. Kleiman and R. Speiser, Enumerative geometry of cuspidal plane cubics, Vancouver Proc., Canad. Math. Soc. Conf. Proc. 6 (1986); Enumerative geometry of nodal plane cubics, Algebraic Geometry (Sundance, 1986), Lecture Notes in Math., vol. 1311, Springer, 1988; Enumerative geometry of non-singular plane cubics (to appear).

[M] S. Maillard, Recherche des caractéristiques des systèmes élémentaires de courbes planes du troisième ordre, Thèses présentées á la Faculté des Sciences de Paris 39 (1871). 
[Sa] G. Sacchiero, Numeri caratteristici delle cubiche piane cuspidali;Numeri caratteristici delle cubiche piane nodali, preprints (1985).

[Sc] H. C. H. Schubert, Kalkül der abzählenden Geometrie (1879), reprinted with an introduction by S. L. Kleiman, Springer-Verlag, 1979.

[St] U. Sterz, Berühungsvervollständigung für ebene Kurven dritter Ordnung I, Beiträge zur Algebra und Geometrie 16 (1983), 45-68, II, 17 (1984), 115-150; III, 20 (1985), 161-184; IV, 21 (1986), 91-108.

[V] I. Vainsencher, Schubert calculus for complete quadrics, Enumerative Geometry and Classical Algebraic Geometry, Progress in Mathematics 24, Birkhäuser, 1982.

[XM] S. Xambó and J. W. Miret, Fundamental numbers of cuspidal cubics; Fundamental numbers of nodal cubics, preprints (1987).

[Z] H. G. Zeuthen, Détermination des caractéristiques des systèmes élémentaires de cubiques, Comptes Rendus des Séances de l'Académie des Sciences 74 (1872), 521-526, 604-607, 726-729.

Department of Mathematics, The University of Chicago, Chicago Illinois 60637 74078

Current address: Department of Mathematics, Oklahoma State University, Stillwater, Oklahoma 